\title{
Maximum helium content of multiple populations in the globular cluster NGC 6752
}

\author{
F. Martins ${ }^{1}$, W. Chantereau ${ }^{2}$, and C. Charbonnel ${ }^{3,4}$ \\ 1 LUPM, Université de Montpellier, CNRS, Place Eugène Bataillon, 34095 Montpellier, France \\ e-mail: fabrice.martins@umontpellier.fr \\ 2 Université de Strasbourg, CNRS, UMR7550, Observatoire Astronomique de Strasbourg, 67000 Strasbourg, France \\ 3 Department of Astronomy, University of Geneva, Chemin Pegasi 51, 1290 Versoix, Switzerland \\ ${ }^{4}$ IRAP, CNRS UMR 5277 \& Université de Toulouse, 14 Avenue Edouard Belin, 31400 Toulouse, France \\ Received 12 March 2021 / Accepted 14 April 2021
}

\begin{abstract}
Context. Multiple populations in globular clusters are usually explained by the formation of stars out of material with a chemical composition that is polluted to different degrees by the ejecta of short-lived, massive stars. But the nature of the "polluters" remains elusive. Different types of stars have been proposed to account for the observed chemical patterns of multiple populations. Among other things, these differ by the amount of helium they spread in the surrounding medium.

Aims. In this study we investigate whether the present-day photometric method used to infer the helium content of multiple populations indeed gives the true value or underestimates it by missing very He-rich, but rare stars. This check is important to discriminate between the different polluter scenarios. We focus on the specific case of NGC 6752.

Methods. We compute atmosphere models and synthetic spectra along isochrones produced for this cluster for a very broad range of He abundances covering the predictions of the different scenarios, including the extreme case of the fast-rotating massive star (FRMS) scenario. We use the same abundances in isochrones and atmosphere models to ensure consistency. We calculate synthetic photometry in HST filters best suited to study the helium content. We subsequently build synthetic clusters with various distributions of stars. We finally determine the maximum helium mass fraction of these synthetic clusters using a method similar to that applied to observational data. In particular, we select nonpolluted and very He-rich stars from the so-called chromosome map.

Results. We re-determine the maximum helium mass fraction $Y$ in NGC 6752, and find a value consistent with published results. We build toy models of clusters with various distributions of multiple populations and ensure that we are able to recover the input maximum $Y$. We then build synthetic clusters with the populations predicted by the FRMS scenario and find that while we slightly underestimate the maximum $Y$ value, we are still able to detect stars much more He-rich than the current observed maximum $Y$. This result still holds even in synthetic clusters that contain less He-rich stars than predicted by the FRMS scenario. It is easier to determine the maximum $Y$ on main sequence stars than on red giant branch stars, but qualitatively the results are unaffected by the sample choice.

Conclusions. We show that in NGC 6752 it is unlikely that stars more He-rich than the current observational limit of about 0.3 (in mass fraction) are present.
\end{abstract}

Key words. globular clusters: general - globular clusters: individual: NGC 6752 - stars: atmospheres - techniques: photometric

\section{Introduction}

Globular clusters (GCs) host multiple populations of stars characterized by different spectroscopic and photometric signatures. Anti-correlations between the abundances of several elements such as $\mathrm{C}-\mathrm{N}, \mathrm{Na}-\mathrm{O}$, and sometimes $\mathrm{Mg}-\mathrm{Al}$ have been reported at the surface of various types of stars, from the main sequence (MS) to the red giant branch (RGB) and the asymptotic giant branch (AGB; e.g., Cohen 1978; Peterson 1980; Sneden et al. 1992; Kraft 1994; Thévenin et al. 2001; Gratton et al. 2007, 2019; Mészáros et al. 2015; Carretta 2015, 2019; Johnson et al. 2016; Pancino et al. 2017; Wang et al. 2017; Masseron et al. 2019). These differences in chemical composition are thought to explain the various sequences observed in color-magnitude diagrams (CMDs) built with specific filters sensitive to atomic and molecular lines of the elements listed above (e.g., Bedin et al. 2004; Piotto et al. 2007; Bowman et al. 2017; Nardiello et al. 2018; Marino et al. 2019). The discovery of these peculiarities (variations in chemical composition and discrete sequences in CMDs) turned GCs from textbook examples of clusters born in a single star forming event into complex structures the origin of which remains elusive.

The standard explanation ${ }^{1}$ to these puzzling properties is the pollution of the original proto-cluster gas by a population of rapidly evolving stars more massive than the present-day GC members. Indeed, the abundance patterns observed are typical outcomes of nucleosynthesis at about $75 \mathrm{MK}$. More precisely, hydrogen burning through the $\mathrm{CNO}$ cycle, together with the $\mathrm{Ne}-\mathrm{Na}$ and the $\mathrm{Mg}-\mathrm{Al}$ chains, fully explains the anti-correlations (Kudryashov \& Tutukov 1988; Denisenkov \& Denisenkova 1990; Prantzos et al. 2007, 2017). Potential polluters experiencing such nucleosynthesis phases are $\sim 5-7.5 M_{\odot}$ AGB stars (e.g., Ventura et al. 2001; Ventura \& D'Antona 2009), massive stars either rotating fast (Decressin et al. 2007a,b), in binary systems (de Mink et al. 2009; Izzard et al. 2013), or in a red supergiant phase (Szécsi \& Wünsch 2019), and super-massive

\footnotetext{
1 Alternative possibilities exist but all rely at least partly on nucleosynthesis products of AGB or massive stars, see Marcolini et al. (2009), Elmegreen (2017).
} 
stars (Denissenkov \& Hartwick 2014). Whatever the nature of the polluter, it should be objects present at early times of the GC history that have spread their nucleosynthesis products in the surrounding gas and out of which the stellar populations we see today were formed. Depending on the degree of mixing of the nucleosynthesis products with pristine gas, a range of chemical composition is predicted for the newly formed stars. The polluters have long disappeared because of their relatively high mass, and only low-mass stars either from the initial population formed out of original proto-GC gas or from the second population formed out of polluted material are still present. We usually refer to these two populations of stars as $1 \mathrm{P}$ (or $1 \mathrm{G}$ ) and $2 \mathrm{P}$ (or $2 \mathrm{G}$ ), standing for first and second population (generation), respectively.

One of the key differences in the predictions of the scenarios that invoke the different polluters listed above is the amount of helium that is inevitably ejected together with other nucleosynthesis products. Indeed, as hot hydrogen-burning is needed to explain the observed abundance patterns, helium is naturally produced and released too. On one hand, the fast-rotating massive star (FRMS) models of Decressin et al. (2007a), developed specifically to reproduce the observations of the GC NGC 6752, predict a wide range of $Y$ for the ejecta, up to $Y=0.8$, and the test case $^{2}$ of binary evolution presented by de Mink et al. (2009) also predicts a wide range of $Y$, up to 0.63 . On the other hand, models of Doherty et al. (2014) indicate that nucleosynthesis in massive AGB stars should produce material with helium mass fraction of about $0.35-0.40$, as a result of second dredge-up. On the other hand, supermassive stars can potentially release material rich in hot hydrogen-burning products but only mildly enriched in helium (Denissenkov \& Hartwick 2014), with a helium mass fraction close to that of the proto-cluster gas in the case where they form through the so-called runaway collision scenario (Gieles et al. 2018).

Measuring the helium surface abundance from spectroscopy is not possible in most GC stars. Only hot horizontal branch (HB) stars display weak He I lines, and these are difficult to model and interpret. However, the helium surface abundance in these stars, with effective temperatures higher than $\sim 11000 \mathrm{~K}$, is affected by atomic diffusion (e.g., Michaud et al. 2008; Quievy et al. 2009). Thus, the measured helium no longer represents the original chemical composition of the stars. The vast majority of GC stars are too cool to display any helium line in their spectra. Nevertheless, helium enrichment is detected in a few hot $\mathrm{HB}$ stars with $\Delta Y$-the difference between the highest $Y$ of $2 \mathrm{P}$ stars and $Y$ of $1 \mathrm{P}$ stars- generally not larger than $\sim 0.1$ (Villanova et al. 2012; Marino et al. 2014), although Pasquini et al. (2011) and Dupree \& Avrett (2013) report a $Y$ difference of up to 0.17 between two HB stars, of NGC 2808 and $\omega$ Cen, respectively.

A change in helium mass fraction affects the internal structure as well as the color and the brightness of stars because the opacity is modified. As a consequence, stars with the same mass, age, and metallicity but different $Y$ have different effective temperatures $\left(T_{\text {eff }}\right.$; the higher $Y$, the higher $T_{\text {eff }}$; e.g., Iben \& Faulkner 1968; Chantereau et al. 2015; Cassisi \& Salaris 2020). Their spectral energy distributions (SEDs) therefore peak at different wavelengths: stars with high $Y$ have more flux at shorter wavelengths, and thus bluer colors. Consequently they are located to the left of stars with smaller $Y$ in

\footnotetext{
2 The study focuses on one binary system made of a $20 M_{\odot}$ primary and a $15 M_{\odot}$ secondary star orbiting each other on a 12-day orbit, and with individual rotation periods synchronized on the orbital period.
}

CMDs (e.g., Sbordone et al. 2011 and Sect. 2.2). Variations in the helium content naturally lead to a widening of classical branches (MS, RGB, HB, AGB) in CMDs (e.g., Rood 1973; Norris et al. 1981; D'Antona \& Caloi 2004; D'Antona et al. 2005; Chantereau et al. 2016). In addition, the associated variations in (among others) $\mathrm{C}, \mathrm{N}$, and $\mathrm{O}$ abundances impact specific colors built with photometric filters encompassing lines sensitive to these elements. This further increases the separation between stars in CMDs, leading to the observed multiple and discrete sequences (e.g., Lardo et al. 2012; Marino et al. 2019).

Comparison of observed and theoretical colors in filters mostly sensitive to $T_{\text {eff }}$, and thus $Y$, has been used to indirectly constrain the amount of helium present at the surface of GC stars (e.g., Piotto et al. 2007; Milone 2015). King et al. (2012) report a maximum helium mass fraction of about $0.39 \pm 0.02$ in $\omega$ Cen based on the analysis of MS stars. Using the Hubble Space Telescope (HST) ultra-violet (UV) survey of GCs (HUGS Piotto et al. 2015; Nardiello et al. 2018), Milone et al. (2018) determined $Y$ in 57 GCs. $\Delta Y$ ranges from nearly 0 to 0.124 , corresponding to a maximum $Y$ of about 0.38 . Milone et al. (2018) also show that $\Delta Y$ correlates with the cluster mass, with higher $Y$ being determined in more massive clusters.

Another indirect constraint on the helium content of GCs comes from the morphology of their HB. Its different shape in clusters with similar general properties, such as M3 and M 13 (e.g., Ferraro et al. 1997), is difficult to explain. A spread in $Y$ of HB stars is a viable possibility (Rood 1973) and quantitative determinations give $\Delta Y$ between 0.02 and 0.15 on the $\mathrm{HB}$, depending on the cluster (Caloi \& D'Antona 2005; Lee et al. 2005; di Criscienzo et al. 2010; Valcarce et al. 2016; Tailo et al. 2016; Denissenkov et al. 2017; VandenBerg \& Denissenkov 2018; Chantereau et al. 2019).

The presence of hot HB stars in GCs of early-type galaxies is also thought to be responsible for the existence of a socalled UV upturn (Greggio \& Renzini 1990). This feature refers to the increase of flux below $\sim 2500 \AA$ in galaxies that no longer form stars and that are made of low-mass MS and post-MS stars. These objects have SEDs that rapidly drop short of about $3500 \AA$. Hot HB stars, such as those seen in Galactic GCs, may explain the UV fluxes. Ali et al. (2018) argued that if such hot HB stars are present because of a high helium content (as suggested by Meynet et al. 2008 for elliptical galaxies), there should be a redshift above which they would no longer be observed, having not yet sufficiently evolved. The position of this transition redshift can be used to constrain $Y$ in hot HB stars. Ali et al. (2018) showed that a value of $\sim 0.45$ would be compatible with the observed disappearance of the UV upturn with redshift.

Thus, modern estimates of $Y$ in GCs indicate that the very helium-rich stars predicted in particular by the FRMS scenario are not detected. However, a key specificity of the FRMS model highlighted by Chantereau et al. (2015) is that such He-rich objects evolve faster and differently compared to more classical stars. In particular, Chantereau et al. (2016) showed that in a GC that would have been formed under the FRMS scenario, the distribution of stars quickly falls as $Y$ increases (at ages typical of GCs, i.e., 9 to $13.5 \mathrm{Gyr}$ ). In practice, there should be little to no He-rich stars on the RGB and AGB of the cluster NGC 6752, for which the models of Chantereau et al. were tailored. This also naturally explains the lack of Na-rich AGB stars in some GCs (Campbell et al. 2013; Wang et al. 2016, 2017), although observations reveal that the presence of 2 P AGB stars can be affected by more than one factor (Wang et al. 2017).

The question therefore naturally arises as to whether the absence of very He-rich stars (i.e., $Y>0.4$ ) is a robust 


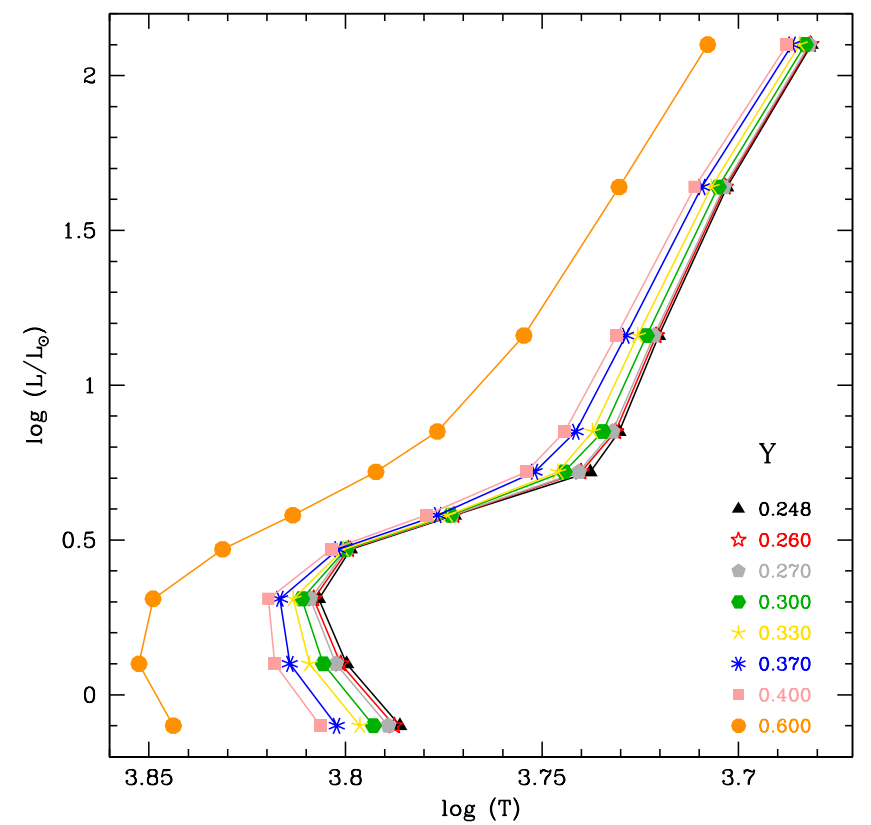

Fig. 1. Hertzsprung-Russell diagram with the isochrones of Chantereau et al. (2015). Different symbols and colors stand for different initial composition, especially different helium mass fraction. Symbols correspond to the points for which atmosphere models have been calculated.

observational fact, or these stars simply escaped detection due to their exceptionally small number. In view of the discriminating nature of the helium content for various scenarios, it is important to investigate this potential issue, which we aim to do in the present study. In practice, we build on the work of Chantereau et al. (2016) to compute synthetic clusters with the predicted distribution of multiple populations for NGC 6752. We compute synthetic spectra consistently with isochrones, produce synthetic photometry and CMDs, and perform determinations of the maximum $Y$ values of these synthetic clusters in order to see if we recover or miss the highly enriched populations predicted by the model of Chantereau et al.

Our paper is organized as follows: Sect. 2 presents the computation of synthetic spectra and photometry. We then discuss the behavior of our models with special emphasis on the effects of surface abundances on colors. We subsequently compare our predicted photometry to observations in various CMDs before moving to the determination of the maximum helium content in NGC 6752 and synthetic clusters in Sect. 3. We discuss our results in Sect. 4 and give our conclusions in Sect. 5.

\section{Spectral synthesis and synthetic photometry}

In this section we first present our computations of synthetic spectra and the associated photometry (Sect. 2.1). We then describe the effects of stellar parameters and surface abundances on both the SEDs and synthetic colors (Sect. 2.2). We build synthetic CMDs and compare them with observations (Sect. 2.3).

\subsection{Method}

We proceeded as in Martins et al. (2020) to compute synthetic photometry. We relied on the evolutionary tracks and isochrones computed by Chantereau et al. (2015) with the code STAREVOL. These models are based on the FRMS scenario
(Decressin et al. 2007a) for the formation of multiple stellar populations in GCs. Each isochrone is characterized by a set of abundances that directly comes from the FRMS predictions. We refer to Chantereau et al. (2015) for details. Each set of abundances is tailored to reproducing $1 \mathrm{P}$ and various $2 \mathrm{P}$ stars in NGC 6752, in which the abundances vary according to different degrees of pollution by FRMS. Figure 1 shows the Hertzsprung-Russell diagram (HRD) with the isochrones we consider in the present work. Each isochrone is labeled according to its helium mass fraction $(Y)$, but we stress that abundances of other elements are also varied (in particular, nitrogen is increased and carbon and oxygen are depleted when $Y$ increases; see Appendix A). We stress that the isochrones have been recomputed for a metallicity of $[\mathrm{Fe} / \mathrm{H}]=-1.53$, which is slightly higher than the value of -1.75 used in Chantereau et al. (2015). This change was made to better match the metallicity of NGC 6752 .

Along each isochrone we computed atmosphere models and synthetic spectra at ten points (see Fig. 1) using the codes ATLAS12 (Kurucz 2014) and SYNTHE (Kurucz 2005). Our computations include the so-called predicted lines, that is, lines for which at least one of the energy levels comes from quantum mechanics calculation and not from laboratory measurements. These lines thus have approximate wavelengths, but their opacities have been shown to be of prime importance to reproducing observed SEDs (Coelho 2014). We adopted a microturbulent velocity of $1 \mathrm{~km} \mathrm{~s}^{-1}$ in all our calculations.

To convert the HRD into CMDs we computed synthetic photometry in the Vegamag system:

$m X=-2.5 \log \left(F_{X} / F_{X}^{\text {Vega }}\right)=-2.5 \log \left(F_{X}\right)+\mathrm{ZP}_{X}^{\text {Vega }}$,

where $m X$ is the magnitude in the $X$ filter and ZP the zero point. The average flux $F_{X}$ over the passband $X$ was calculated according to Bohlin et al. (2014):

$F_{X}=\frac{\int \lambda F_{\lambda} R_{X} \mathrm{~d} \lambda}{\int \lambda R_{X} \mathrm{~d} \lambda}$,

where $R_{X}$ is the transmission curve of filter $X^{3}$. The zero point $\mathrm{ZP}_{X}^{\text {Vega }}$ in Eq. (1) was calculated using the Vega STScI reference spectrum $^{4}$ and the appropriate transmission curve.

Finally, for comparison with observations, we assumed a distance modulus of 13.18 for NGC 6752 based on the Gaia DR2 determination (Gaia Collaboration 2018). This value is consistent with those found by Harris $(1996 ; 13.19)$, Renzini et al. (1996; 13.05), and Gratton et al. (2003; 13.24). Prior to synthetic photometry calculations, we reddened our SEDs using a color excess $E(B-V)=0.060$ and $A_{V}=3.2$, adopting the extinction law of Cardelli et al. (1989). This choice best reproduces the turn-off (TO) and sub-giant region in the $\mathrm{m} 814 \mathrm{~W}$ (m606W-m814W) CMD (see for instance Fig. 6). This is slightly larger than the value of $0.046 \pm 0.005$ reported by Gratton et al. (2005) but close to the value of Schlegel et al. (1998): 0.056.

\subsection{Spectral energy distributions}

In this section we describe the effects of chemical composition on the SED. The goal is to identify spectral regions that are

\footnotetext{
3 Transmission curves were retrieved from the Spanish VO http:// svo2.cab.inta-csic.es/svo/theory/fps3/

4 File alf_lyr_stis_010.fits from ftp://ftp.stsci.edu/cdbs/ current_calspec/
} 


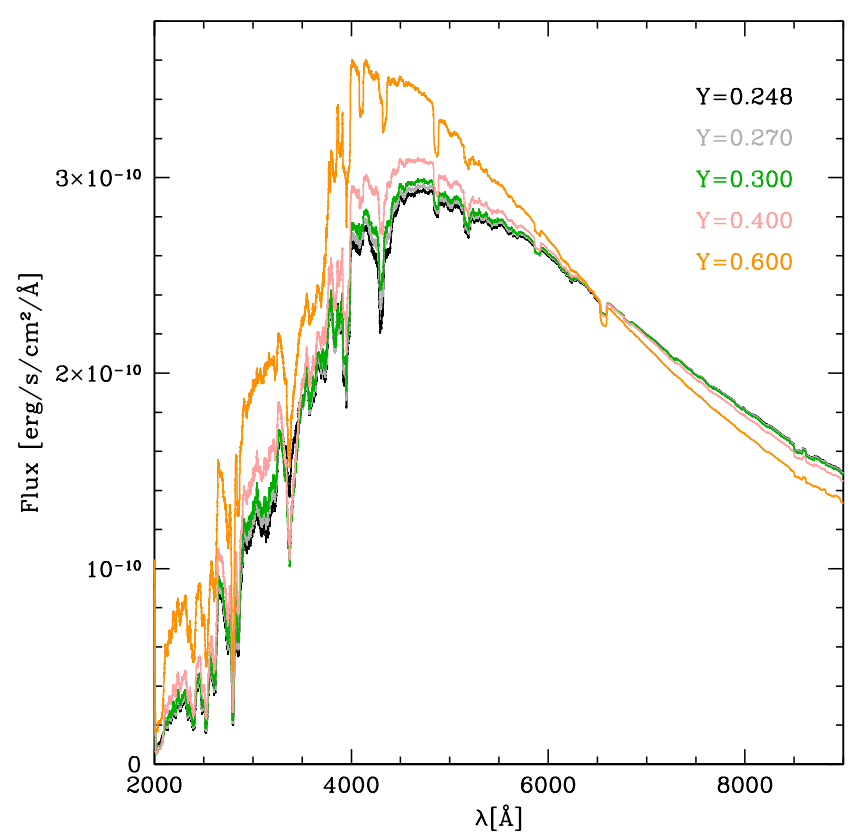

Fig. 2. Spectral energy distributions of some of the models with $\log \frac{L}{L_{\odot}}=0.85$ but different chemical compositions (and thus different $T_{\text {eff }}$ and $\log g$ ). Different colors correspond to different models, identified by their helium mass fraction.

affected by certain species. We refer to Sbordone et al. (2011) or Milone et al. (2020), among other recent studies, for similar descriptions.

Figure 2 shows a selection of models at the bottom of the RGB, with luminosities equal to $10^{0.85} L_{\odot}$ (see Fig. 1). These spectra have different $T_{\text {eff }}$, surface gravities and chemical compositions (see Table A.1). Because of the higher $T_{\text {eff }}$ in higher $Y$ models, the SED peak is shifted towards shorter wavelengths. In the optical region, the slope of the SED becomes steeper (faster decline with wavelength) which translates into bluer colors. Variations in individual abundances of light elements (CNO) also changes the strength of absorption lines, mostly below $4500 \AA$.

To better separate the effects of light elements and helium on the SED, we show in Fig. 3 examples of spectra in which the abundances of $\mathrm{C}, \mathrm{N}$, and $\mathrm{O}$ have been varied by a factor of three, all other parameters being kept constant. A reduction of the carbon abundance translates into a weaker $\mathrm{CH}$ absorption between 4200 and $4400 \AA$. Conversely, an increase in the nitrogen content leads to a stronger $\mathrm{NH}$ absorption at 3300-3500 A. The $\mathrm{CN}$ band around $3900 \AA$ is less affected. The $\mathrm{OH}$ absorption between 2800 and $3300 \AA$ reacts to a change in the oxygen content. Consequently, photometry in the HST filters $F 275 \mathrm{~W}$ and $F 336 W$ is affected by $\mathrm{C}, \mathrm{N}$, and $\mathrm{O}$ abundances (Sbordone et al. 2011; Milone et al. 2018; Cassisi \& Salaris 2020). These filters have been used to build the so-called super color $\mathrm{C}_{410}$ $=(\mathrm{m} 275 \mathrm{~W}-\mathrm{m} 336 \mathrm{~W})-(\mathrm{m} 336 \mathrm{~W}-\mathrm{m} 410 \mathrm{M})($ Milone et al. 2013). This photometric diagnostic has been shown to be a powerful tool for separating multiple populations (see also following section). Filters $F 395 N, F 467 M, F 606 W, F 814 W$ and to some extent $F 410 M$ are relatively insensitive to variations in $\mathrm{C}, \mathrm{N}$, and $\mathrm{O}$ abundances. An important result of Fig. 3 is that $\mathrm{C}, \mathrm{N}$, and $\mathrm{O}$ abundances do not affect the global shape of the SED, but only specific wavelength regions that include molecular bands (Milone et al. 2013; Dotter et al. 2015).
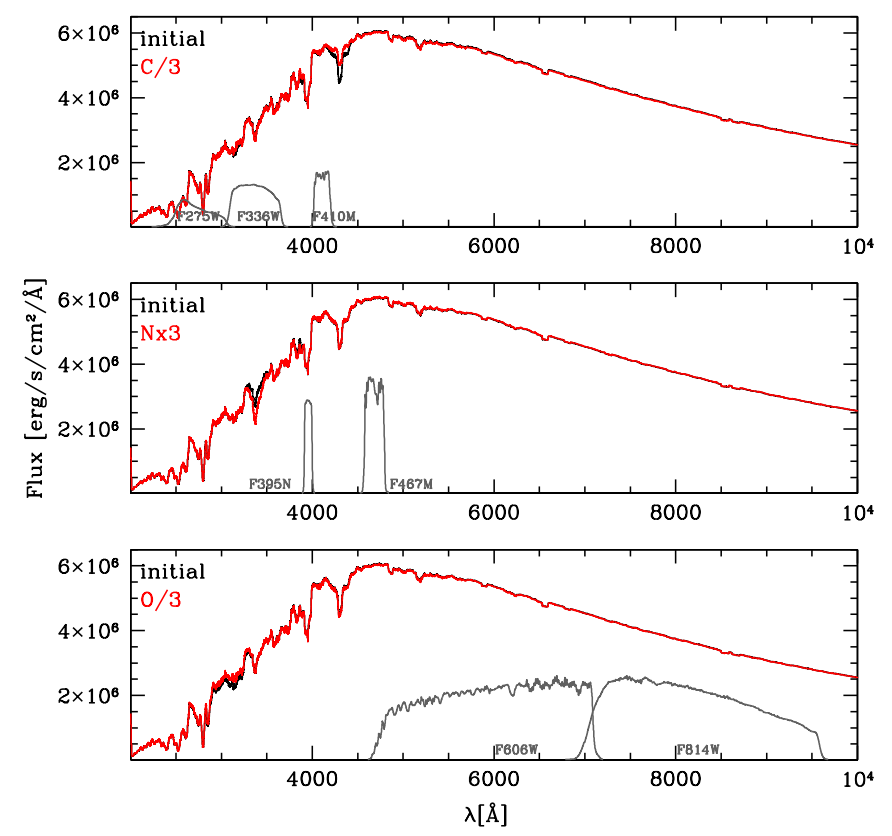

Fig. 3. Spectral energy distribution of two models with similar parameters except the $\mathrm{C}, \mathrm{N}$, and $\mathrm{O}$ abundances: the red line shows a model with $\mathrm{C} / \mathrm{H}$ reduced by a factor three (top panel), N/H increased by a factor three (middle), and $\mathrm{O} / \mathrm{H}$ reduced by a factor three (bottom) compared to the initial model in black. The other parameters are: $T_{\text {eff }}=5375 \mathrm{~K}$, $\log g=3.37, Y=0.248$. The gray solid lines indicate the transmission curves of the HST WFC3/UVIS F275W, F336W, F395N, F410M, $F 467 M$, and ACS/WFC $F 606 W$ and $F 814 W$.

To further quantify the effect of chemical composition (and in particular of $Y$ ) on the SED, in Fig. 4 we compare the SEDs of three models with the same luminosity but different chemical composition. We focus on the models with $L=10^{0.85} L_{\odot}$. We have chosen the models of the $Y=0.248$ and $Y=0.400$ sequence, to which we add a third model with the effective temperature and gravity of the $Y=0.248$ sequence, but the chemical composition of the $Y=0.400$ sequence. Hence, we are able to disentangle the effect of chemical composition on the effective temperature and $\log g$ on one side, and on the SED on the other side. Comparing the two models with $T_{\text {eff }}=5375 \mathrm{~K}$, we find the same trends as the ones drawn from Fig. 3. It is mainly the $\mathrm{CH}$, $\mathrm{CN}, \mathrm{NH}$ and $\mathrm{OH}$ bands that are affected. The general shape of the SED is otherwise relatively similar in the two models. Hence, models with different helium content but similar $T_{\text {eff }}$ and surface gravity have very similar SEDs, except in regions containing lines involving $\mathrm{C}, \mathrm{N}$, and $\mathrm{O}$. If we now compare the two models with the same composition but different effective temperature and surface gravity, we see a major modification of the SED. The hottest model has more (less) flux blueward (redward) of $6000 \AA$. This is mainly governed by the change in $T_{\text {eff }}$ which itself is due to the different helium content. Figure 5 shows the temperature structure in the three models. Changing only the chemical composition leads to a higher temperature in the inner atmosphere, but a similar structure in the outer parts. This effect is entirely dominated by the difference in the helium content: computing a model with $T_{\text {eff }}=5375 \mathrm{~K}, \log g=3.37$, and the helium content of the $Y=0.400$ sequence but the abundances of all elements heavier than He from the $Y=0.248$ sequence leads to a temperature structure which is almost indistinguishable from that of the green model in Fig. 5. If we now change both the chemical composition and the effective temperature/surface gravity (red 


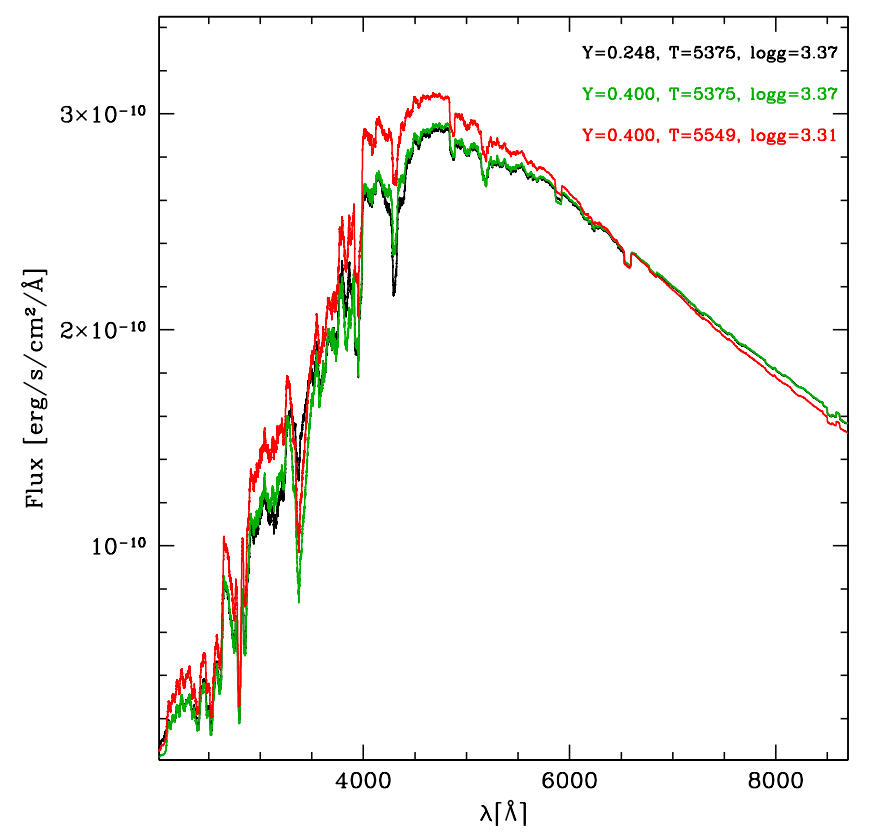

Fig. 4. Spectral energy distribution of three models with $\log \frac{L}{L_{\odot}}=0.85$ one with $Y=0.248, T_{\text {eff }}=5375$ and $\log g=3.37$ (black line); one with $Y=0.400$ and the same $T_{\text {eff }}$ and $\log g$ (green line); and the model with $Y=0.400$ and the associated $T_{\text {eff }}$ and $\log g(5549 \mathrm{~K}$ and 3.31 respectively, red line).

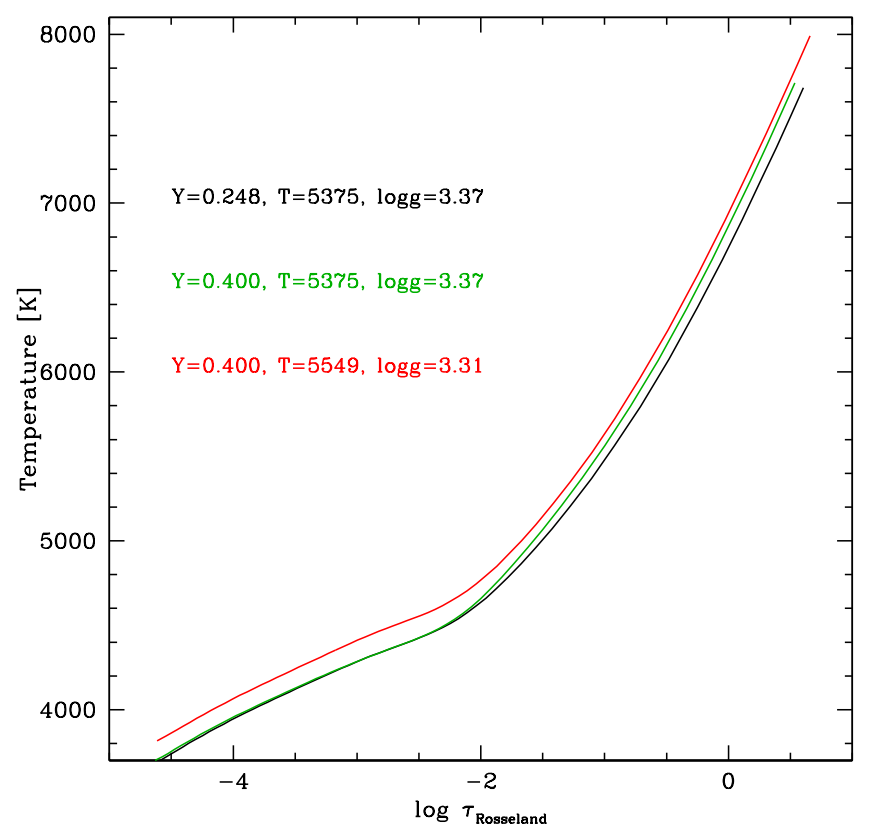

Fig. 5. Temperature as a function of Rosseland optical depth in the three models leading to the SEDs shown in Fig. 2.

model), we obtain a global increase in the temperature at all depths in the atmosphere, which translates into a change of the SED.

From these comparisons we conclude that the global shape of the SED is mainly sensitive to the effective temperature which itself depends critically on the helium content and its effect on opacities. The abundances of heavier elements affect certain portions of the SED but not its global shape. Variations in $Y$ thus affect colors sensitive to the global shape of the SED. The filters $F 395 N, F 410 M, F 606 W$, and $F 814 W$ should be relatively free of contamination by absorption lines of light elements, and can therefore be used to study the helium content (Milone et al. 2013, 2018; Dotter et al. 2015; Cassisi \& Salaris 2020).

\subsection{Comparison with observed CMDs}

In this section we compare our synthetic photometry to the observed CMDs of NGC 6752. We use the data of Milone et al. (2013) and of the HUGS survey (Piotto et al. 2015; Nardiello et al. 2018). The goal is to see if synthetic colors reproduce observations and to identify potential failures.

The bottom-right panel of Fig. 6 shows the m $814 \mathrm{~W}$ versus (m606W-m814W) CMD. Taking the $Y=0.248$ isochrone as a reference, we see that on average our synthetic photometry is able to reproduce the shape of the observed sub-giant branch and the bottom of the RGB. More specifically, the synthetic isochrone is located near the red part of the envelopes that define these branches, which are broadened because of intrinsic dispersion and the presence of multiple populations. The synthetic isochrone reproduces the TO relatively well. On the upper RGB, the synthetic isochrone appears slightly too red compared to the observations, which could be due to the fact that the stellar evolution models were computed with an atmosphere treated in the gray, plane-parallel, and Eddington approximations.

Figure 6 shows several CMDs involving filters $F 275 W$, $F 336 W, F 410 M, F 467 M$ and $F 814 W$. These filters are sensitive to the chemical composition as described in Sect. 2.2. Focusing on the $Y=0.248$ isochrone we find, in general, relatively good qualitative agreement with observations. We note that in the $\mathrm{m} 814 \mathrm{~W}$ versus $(\mathrm{m} 410 \mathrm{M}-\mathrm{m} 814 \mathrm{~W})$ diagram, the synthetic isochrone is located slightly too much to the red on the RGB (at least when compared to the m814W-(m275W-m814W) and $\mathrm{m} 814 \mathrm{~W}-(\mathrm{m} 336 \mathrm{~W}-\mathrm{m} 814 \mathrm{~W}) \mathrm{CMDs})$. In the (m336W-m814W) color the $Y=0.248$ isochrone is located roughly in the middle of the observed branch, for reasons that are explained in the following paragraph. The observed RGB is redder than the synthetic isochrone in the $\mathrm{m} 814 \mathrm{~W}$ versus $\mathrm{C}_{410}$ diagram. This is likely the result of the small offsets seen in other CMDs that are amplified by the super color. All these (relatively) small offsets between observations and synthetic isochrones are due to different photometric calibrations between observations and synthetic colors and to limitations in the stellar evolution and spectral modeling. This likely has a small impact on the determination of the helium content of multiple populations, because only relative color differences are used and not absolute ones. However, it is important to keep in mind that full consistency is not achieved between observations and synthetic photometry.

Increasing $Y$ globally leads to a shift of all isochrones to the left because of the increased $T_{\text {eff }}$. An exception is the $\mathrm{m} 814 \mathrm{~W}-(\mathrm{m} 336 \mathrm{~W}-\mathrm{m} 814 \mathrm{~W}) \mathrm{CMD}$ where the isochrones move first to the right $(Y=0.260$ to $Y=0.300)$ and then move back to the blue from $Y=0.300$ to $Y=0.600$. This is caused by the strong sensitivity of the $F 336 \mathrm{~W}$ filter to the nitrogen abundance (see Sect. 2.2). The $\mathrm{N}$ abundance increases rapidly when $Y$ increases, which causes a deepening of the NH absorption band. Consequently, the $(\mathrm{m} 336 \mathrm{~W}-\mathrm{m} 814 \mathrm{~W})$ color is redder. At the same time, $T_{\text {eff }}$ increases because of the higher helium content. But at first, the effect of nitrogen is stronger, leading to a redder color. When $Y$ reaches $\sim 0.300$, the nitrogen increase is not sufficient to counter-balance the effect of the higher $T_{\text {eff }}$ and the colors become bluer again. This effect, specific to the $F 336 \mathrm{~W}$ filter, is not seen in the TO region of the CMD because at the corresponding $T_{\text {eff }}$ there is no molecular $\mathrm{NH}$ band in the spectra of the stars investigated here. 

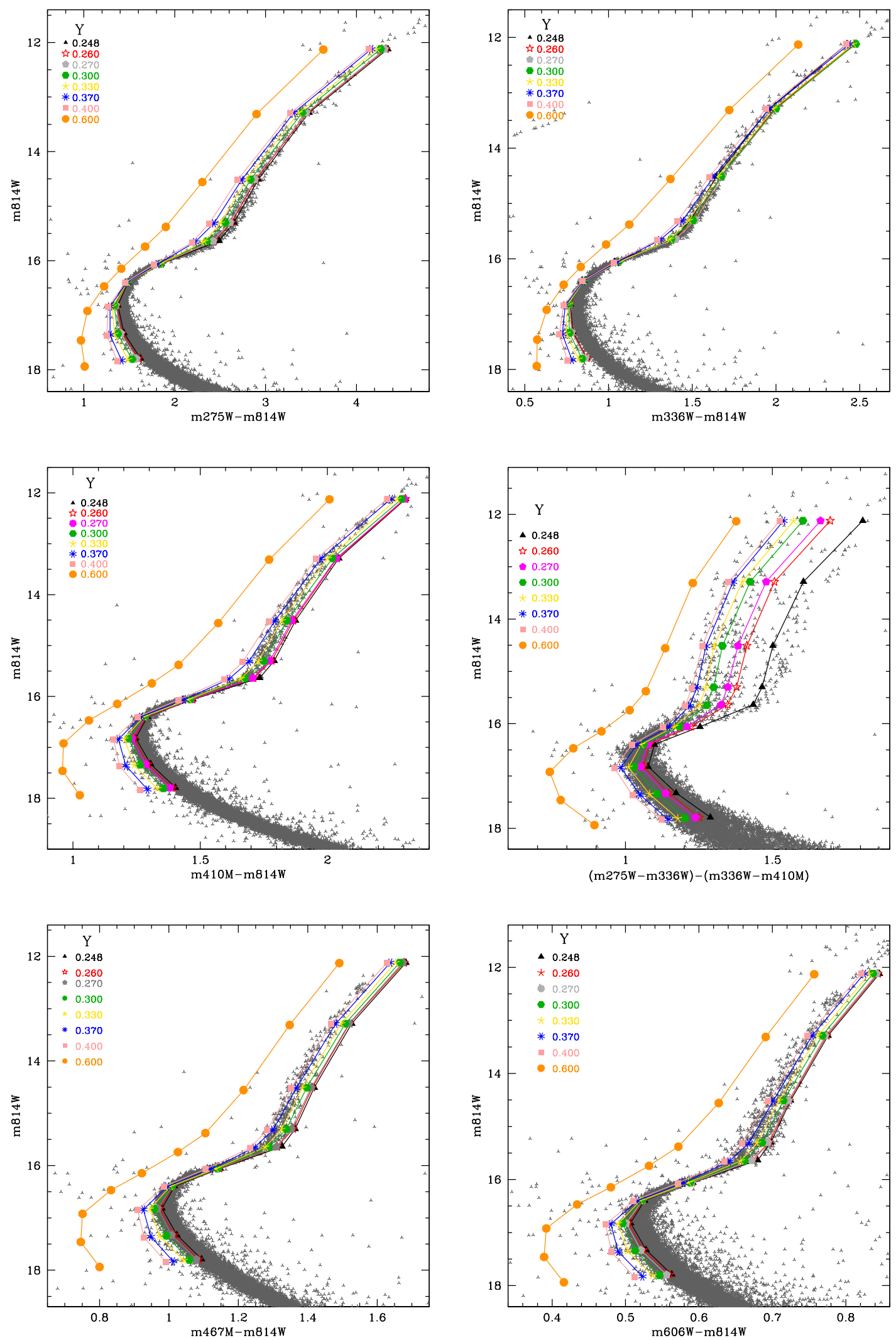

Fig. 6. Color-magnitude diagrams: In all panels, the ordinate axis is the magnitude in the ACS $F 814 W$ filter. The abscissa axis is the difference between magnitude in the $X$ filter and the magnitude in the ACS $F 814 W$ filter, where $X$ is the WFC3 F275W filter (top left panel), WFC3 $F 336 \mathrm{~W}$ filter (top right panel), WFC3 F410M filter (middle left panel), WFC3 F467M filter (bottom left panel), and ACS $606 \mathrm{~W}$ (bottom right panel). Middle right panel: abscissa axis is the color difference $(\mathrm{m} 275 \mathrm{~W}-\mathrm{m} 336 \mathrm{~W})-(\mathrm{m} 336 \mathrm{~W}-\mathrm{m} 410 \mathrm{M})$. In all panels, different symbols and colors correspond to models with different chemical composition, tagged by their helium content $(Y)$. Gray points correspond to the photometric data of Milone et al. (2013), except in the bottom right panel where they are from the HUGS survey (Piotto et al. 2015; Nardiello et al. 2018). 
Having presented and described our synthetic photometry of NGC 6752, we now turn to the main question tackled by this study: the maximum helium content.

\section{Maximum helium content}

In this section, we investigate whether the current estimates of the maximum helium content of stars of the second population are true values or lower limits. More precisely, we study the possibility that highly enriched stars could be missed when studying multiple populations with HST photometry.

\subsection{Helium in NGC 6752}

In a first step, we re-determine the maximum helium mass fraction difference in NGC 6752. We use a similar method to that of Milone et al. (2018) which we describe below. Briefly, we select the extreme populations, that is, the least and most chemically enriched ones, from the chromosome map (Milone et al. 2017). We then estimate the helium mass fraction difference between these two populations in various CMDs using the theoretical isochrones and synthetic photometry presented in Sects. 2.1 and 2.2.

To build the chromosome map we first create the m814W versus $(\mathrm{m} 275 \mathrm{~W}-\mathrm{m} 814 \mathrm{~W})$ and $\mathrm{m} 814 \mathrm{~W}$ versus $\mathrm{C}_{410}$ diagrams shown in the top panels of Fig. 7. We subsequently define the red and blue so-called fiducial lines that bracket the distribution of stars along the RGB. These lines are defined manually by selecting points along the red and blue envelopes and by applying a spline function over the selected points. The width of the RGB is set to the difference between the two fiducial lines at a magnitude of 14.9 , corresponding to $2.0 \mathrm{mag}$ above the TO, in agreement with the definition of Milone et al. (2017). For each star on the RGB in the m814W versus $(\mathrm{m} 275 \mathrm{~W}-\mathrm{m} 814 \mathrm{~W})$ and $\mathrm{m} 814 \mathrm{~W}$ versus $\mathrm{C}_{410}$ diagrams, the quantities $\Delta(\mathrm{m} 275 \mathrm{~W}-\mathrm{m} 814 \mathrm{~W})$ and $\Delta\left(\mathrm{C}_{410}\right)$ are calculated according to Eqs. (1) and (2) of Milone et al. (2017). We select stars with m814W magnitudes between 15.8 and 12.8 (dashed lines in Fig. 7) to cover the bottom of the RGB but avoid its part above the bump in the luminosity function where internal mixing can affect surface chemical composition (e.g., Briley et al. 1990; Shetrone 2003; Charbonnel \& Zahn 2007; Lind et al. 2009; Henkel et al. 2017). The resulting chromosome map is shown in the bottom panel of Fig. 7.

Following the concept of Milone et al. (2017), we manually select the extreme $1 \mathrm{Pe}$ and $2 \mathrm{Pe}$ stars from the chromosome map by defining boxes respectively near the right-most and leftmost parts of the stars' distribution, as illustrated in Fig. 7. We then study the color (and thus $Y$ ) differences between these two extreme populations in three CMDs: $\mathrm{m} 814 \mathrm{~W}$ versus (m395N$\mathrm{m} 814 \mathrm{~W}), \mathrm{m} 814 \mathrm{~W}$ versus $(\mathrm{m} 410 \mathrm{M}-\mathrm{m} 814 \mathrm{~W})$, and $\mathrm{m} 814 \mathrm{~W}$ versus (m467M-m814W). According to Sect. 2.3 and Milone et al. (2018) the colors $(\mathrm{m} 395 \mathrm{~N}-\mathrm{m} 814 \mathrm{~W}),(\mathrm{m} 410 \mathrm{M}-\mathrm{m} 814 \mathrm{~W})$, and (m467M-m814W) depend almost exclusively on the helium content at the metallicity of NGC 6752. In addition, the color difference is the largest for the colors that involve filters located at sufficiently blue wavelengths to show sensitivity to effective temperature -and thus $Y$-variations (see Fig. 2 and middle panel of Fig. 5 of Milone et al. 2018).

Figure 8 shows the $\mathrm{m} 814 \mathrm{~W}$ versus $(\mathrm{m} 395 \mathrm{~N}-\mathrm{m} 814 \mathrm{~W})$ diagram for the $1 \mathrm{Pe}$ and $2 \mathrm{Pe}$ stars (see Appendix B for the other CMDs). Building on Milone et al. (2018) we define new fiducial lines along the two populations. We divide each distribution into $\mathrm{m} 814 \mathrm{~W}$ bins of $0.2 \mathrm{mag}$ in width. In each bin, we calculate the
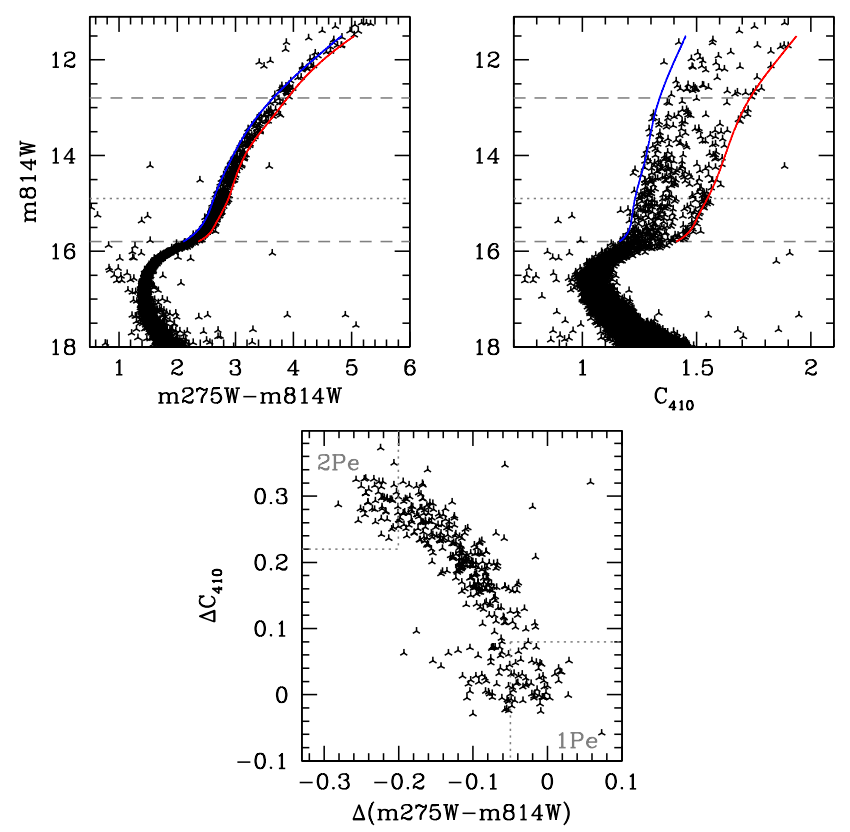

Fig. 7. Top left panel: $\mathrm{m} 814 \mathrm{~W}$ versus $(\mathrm{m} 275 \mathrm{~W}-\mathrm{m} 814 \mathrm{~W}) \mathrm{CMD}$ of NGC 6752. Top right panel: $\mathrm{m} 814 \mathrm{~W}$ versus $\mathrm{C}_{410}$ diagram. In both panels, the red and blue solid lines are the fiducial lines along the RGB. The horizontal dashed lines indicate the magnitude bin considered to build the chromosome map shown in the bottom panel. The horizontal dotted line marks the m814W value at which the width of the RGB is measured (see text). Bottom panel: chromosome map $\left(\Delta\left(\mathrm{C}_{410}\right)\right.$ versus $\Delta(\mathrm{m} 275 \mathrm{~W}-\mathrm{m} 814 \mathrm{~W}))$ on the RGB. The dotted lines define boxes in which stars are considered either $1 \mathrm{Pe}$ (bottom right) or $2 \mathrm{Pe}$ (top left).

median $\mathrm{m} 814 \mathrm{~W}$ and $(\mathrm{m} 395 \mathrm{~N}-\mathrm{m} 814 \mathrm{~W})$. We subsequently perform a boxcar averaging, replacing each median point by the average of its three closest neighbours. This gives the filled circles in Fig. 8. Finally, we run a spline function over these new points to obtain the fiducial lines for each of the two extreme populations. The color difference between these lines is estimated at six m814W magnitudes: 15.2, 14.9, 14.6, 14.3, 14.0, and 13.7. We do not consider brighter stars for reasons that will be explained further below. Appendix B shows the CMDs using the $F 410 M$ and $F 467 M$ filters.

Once obtained, this set of six color differences is compared to theoretical values in Fig. 9. The latter are calculated from our synthetic photometry, including distance and extinction corrections appropriate for NGC 6752 as described in Sect. 2.1. For each m814W magnitude, we determine the $Y$ difference between the isochrones that match the $(\mathrm{m} 395 \mathrm{~N}-\mathrm{m} 814 \mathrm{~W})$ color difference determined from observations. We do this for the six selected m814W magnitudes and finally average the six determinations to yield the final $Y$ difference. The standard deviation is taken as the uncertainty in this measurement. We perform this process for the three selected colors. The results are gathered in Table 1. They are broadly consistent with the determination of Milone et al. (2018) who quote a maximum $Y$ difference of $0.042 \pm 0.004$. We note that our uncertainties are much larger. We tested the effect of varying the size of the boxes to select the $1 \mathrm{Pe}$ and $2 \mathrm{Pe}$ stars in the chromosome map. Increasing the size of $0.2 \mathrm{mag}$ does not affect the results. However, reducing the size (i.e., selecting fewer points, but at even more extreme positions) translates into an increase in $\Delta Y$ by between 0.015 and 0.020 . However, at the same time, the uncertainties also increase by the same amount because of the reduced number of stars. 


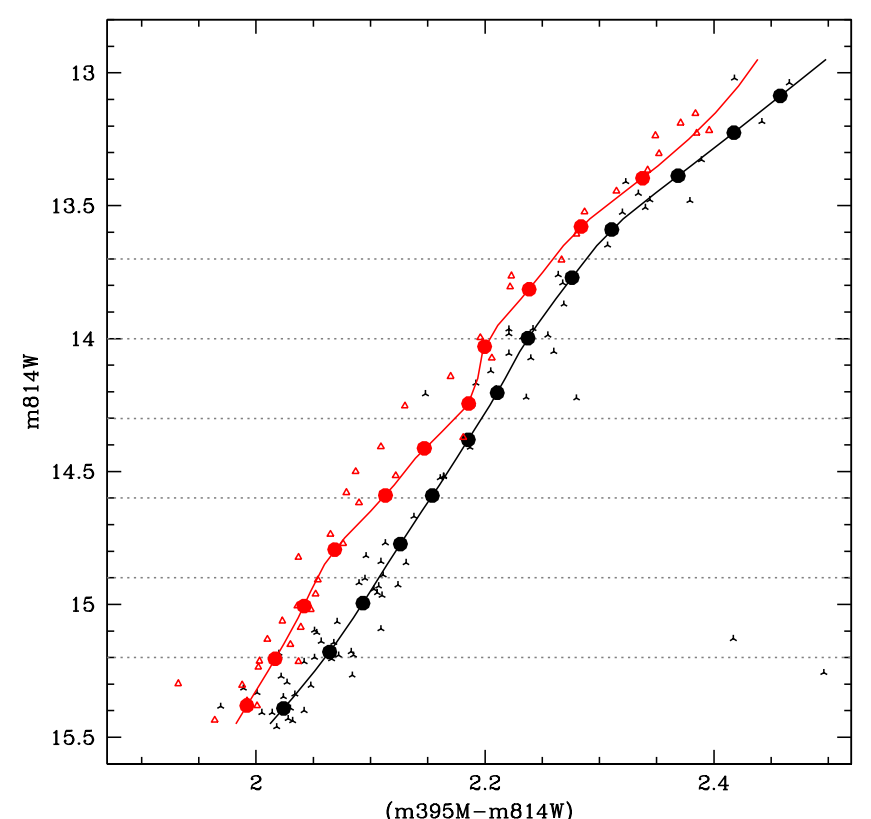

Fig. 8. $m 814 \mathrm{~W}$ versus (m395N-m814W) CMD for the 1 Pe (black) and 2Pe (red) populations selected in the chromosome map of Fig. 7. The filled circles are the points used to define the fiducial lines, which themselves are shown by the solid lines. See text for details. The horizontal dotted lines indicate the $\mathrm{m} 814 \mathrm{~W}$ magnitudes at which the color difference between the $2 \mathrm{Pe}$ and $1 \mathrm{Pe}$ fiducial lines is determined.

In anticipation of the following section, in Fig. 10 and Table 1 we present the analysis of extreme populations on the MS of NGC 6752. The details of the analysis are presented in Sect. 3.2.3. The results indicate that the maximum helium mass fraction reported by Milone et al. (2018) based on the RGB is also recovered for MS stars. These results are further discussed in Sect. 4.

\subsection{Synthetic clusters}

In a second step, we build synthetic clusters to investigate whether or not we underestimate the maximum helium difference between extreme populations.

We first start by building synthetic CMDs. In practice, we draw artificial points along the isochrones in the m814W versus $(\mathrm{m} 275 \mathrm{~W}-\mathrm{m} 814 \mathrm{~W}), \mathrm{m} 814 \mathrm{~W}$ versus $(\mathrm{m} 336 \mathrm{~W}-\mathrm{m} 814 \mathrm{~W}), \mathrm{m} 814 \mathrm{~W}$ versus $(\mathrm{m} 410 \mathrm{M}-\mathrm{m} 814 \mathrm{~W}), \mathrm{m} 814 \mathrm{~W}$ versus $(\mathrm{m} 395 \mathrm{~N}-\mathrm{m} 814 \mathrm{~W})$, and $\mathrm{m} 814 \mathrm{~W}$ versus (m467M-m814W) diagrams (see Fig. 6). We assume a magnitude distribution in the $F 814 \mathrm{~W}$ filter similar to that of NGC 6752 (see Fig. 11). We build clusters made of 9000 stars, ensuring a consistent number of stars on the RGB compared to NGC 6752.

For each m814W magnitude drawn along the isochrone (in each diagram) we add a correction to the color ( $x$-axis in each diagram) to introduce a dispersion on the theoretical isochrone. We estimate the correction in the following way. We retrieve photometry of NGC 6752 from the HUGS survey ${ }^{5}$ and build the $\mathrm{m} 814 \mathrm{~W}$ versus $(\mathrm{m} 275 \mathrm{~W}-\mathrm{m} 814 \mathrm{~W}), \mathrm{m} 814 \mathrm{~W}$ versus $(\mathrm{m} 336 \mathrm{~W}-\mathrm{m} 814 \mathrm{~W})$, and $\mathrm{m} 814 \mathrm{~W}$ versus $(\mathrm{m} 435 \mathrm{~W}-\mathrm{m} 814 \mathrm{~W})$ diagrams. We select stars at m814W magnitudes of 14.9 and 16.5 (in a bin of size $0.2 \mathrm{mag}$ ). For these subgroups of stars and for each color, $(\mathrm{m} 275 \mathrm{~W}-\mathrm{m} 814 \mathrm{~W}),(\mathrm{m} 336 \mathrm{~W}-\mathrm{m} 814 \mathrm{~W})$, and $(\mathrm{m} 435 \mathrm{~W}-\mathrm{m} 814 \mathrm{~W})$, we take the standard deviation with respect

\footnotetext{
5 https://archive.stsci.edu/prepds/hugs/
}
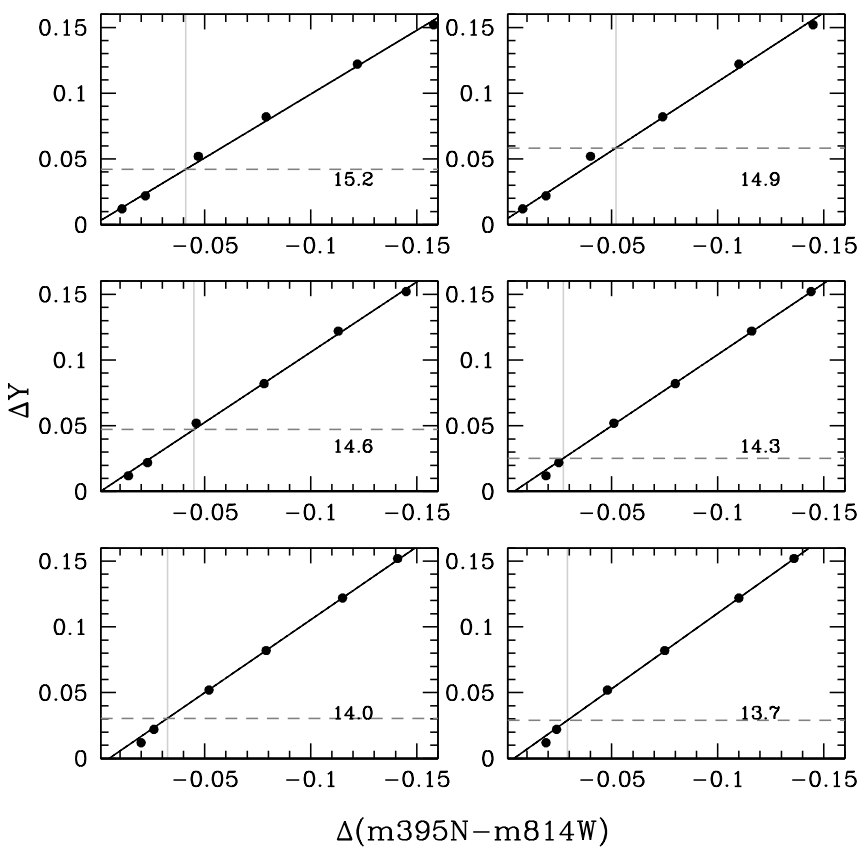

Fig. 9. Determination of the helium mass fraction difference between $1 \mathrm{Pe}$ and $2 \mathrm{Pe}$ stars for the six magnitudes (see labels in panels) defined in Fig. 8. For each panel the filled circles are the theoretical values calculated from our isochrones and synthetic photometry. The black solid line is a linear regression to these values. The vertical gray line highlights the measured color difference between $1 \mathrm{Pe}$ and $2 \mathrm{Pe}$ fiducial lines. The horizontal dashed line indicates the corresponding $Y$ difference read from the black solid line.

Table 1. Difference in the helium mass fraction $Y$ between the $1 \mathrm{Pe}$ and $2 \mathrm{Pe}$ populations, for different colors and for the RGB and MS stars.

\begin{tabular}{lc}
\hline \hline Color & $\Delta Y$ \\
\hline RGB & \\
$(\mathrm{m} 395 \mathrm{~N}-\mathrm{m} 814 \mathrm{~W})$ & $0.039 \pm 0.013$ \\
$(\mathrm{~m} 410 \mathrm{M}-\mathrm{m} 814 \mathrm{~W})$ & $0.052 \pm 0.011$ \\
$(\mathrm{~m} 467 \mathrm{M}-\mathrm{m} 814 \mathrm{~W})$ & $0.068 \pm 0.025$ \\
\hline MS & \\
$(\mathrm{m} 395 \mathrm{~N}-\mathrm{m} 814 \mathrm{~W})$ & $0.042 \pm 0.004$ \\
$(\mathrm{~m} 410 \mathrm{M}-\mathrm{m} 814 \mathrm{~W})$ & $0.047 \pm 0.004$ \\
$(\mathrm{~m} 467 \mathrm{M}-\mathrm{m} 814 \mathrm{~W})$ & $0.049 \pm 0.049$ \\
\hline
\end{tabular}

to the median point as representative of the dispersion on the RGB and MS. We subsequently use these values to introduce a dispersion in the synthetic colors. For that we randomly draw a color correction by means of a Gaussian distribution centered on zero and characterized by the standard deviation determined above. In the absence of photometric error for the $\mathrm{m} 395 \mathrm{~N}, \mathrm{~m} 410 \mathrm{M}$, and $\mathrm{m} 467 \mathrm{M}$ magnitudes, we assume the dispersion in the colors $(\mathrm{m} 395 \mathrm{~N}-\mathrm{m} 814 \mathrm{~W}),(\mathrm{m} 410 \mathrm{M}-\mathrm{m} 814 \mathrm{~W})$, and $(\mathrm{m} 467 \mathrm{M}-\mathrm{m} 814 \mathrm{~W})$ is the same as in $(\mathrm{m} 435 \mathrm{~W}-\mathrm{m} 814 \mathrm{~W})$. We perform this process for all isochrones, that is, for all chemical compositions. We finally end up with a series of synthetic CMDs that are used to build synthetic clusters.

\subsubsection{Two chemical compositions}

We build synthetic clusters by mixing populations of different chemical composition. We start with a cluster made up 

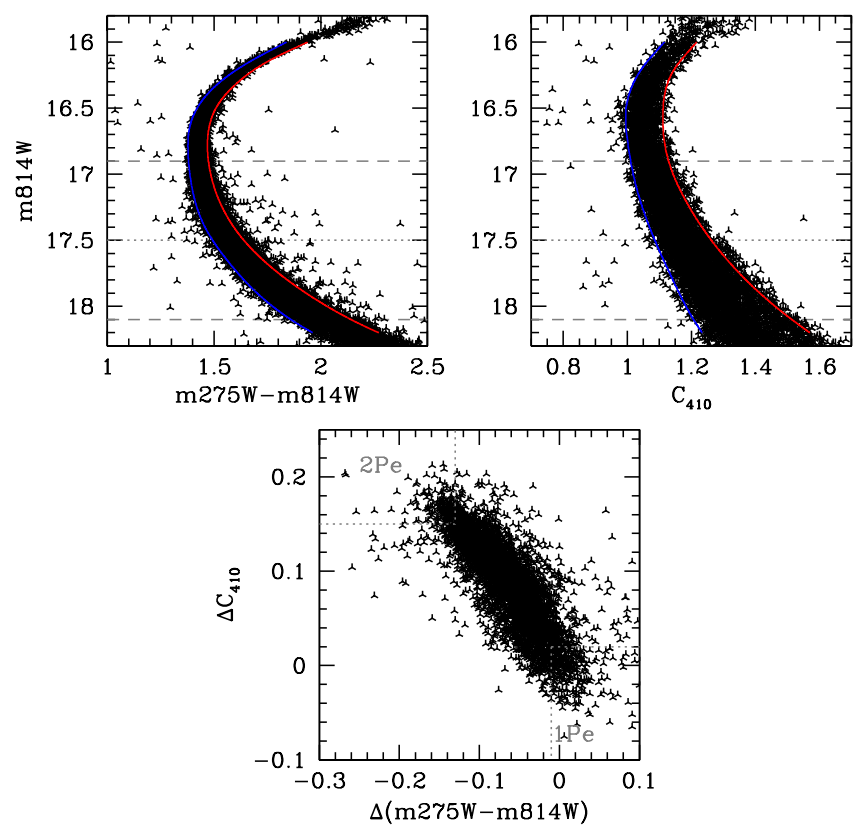

Fig. 10. Same as Fig. 7 but for the MS of NGC 6752.

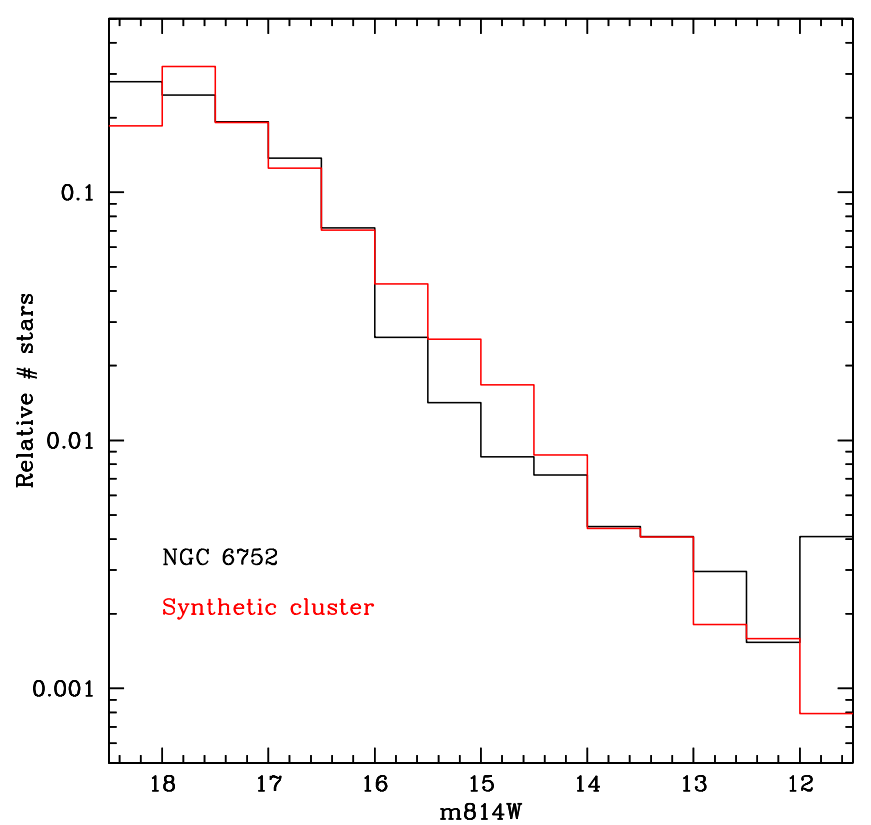

Fig. 11. Distribution of stars according to their m814W magnitude in NGC 6752 (black) and in a typical synthetic cluster (red). The latter distribution results from random sampling using a Gaussian distribution.

of one-third stars with $Y=0.248$, and two-thirds stars with $Y=0.330$. For the $\mathrm{m} 814 \mathrm{~W}$ versus $(\mathrm{m} 275 \mathrm{~W}-\mathrm{m} 814 \mathrm{~W}) \mathrm{CMD}$, we therefore select 3000 stars from $Y=0.248$ isochrone in the synthetic m814W versus $(\mathrm{m} 275 \mathrm{~W}-\mathrm{m} 814 \mathrm{~W})$ just created, and 6000 stars from the $Y=0.330$ isochrone. We repeat the process for all five diagrams. We then apply the same method as described in Sect. 3.1 to determine the maximum helium mass fraction difference in the synthetic cluster. We know by construction that it is equal to 0.082 . We find that $\Delta Y=0.081 \pm 0.002$, $0.079 \pm 0.002$, and $0.070 \pm 0.015$ using the $(\mathrm{m} 395 \mathrm{~N}-\mathrm{m} 814 \mathrm{~W})$, $(\mathrm{m} 410 \mathrm{M}-\mathrm{m} 814 \mathrm{~W})$, and $(\mathrm{m} 467 \mathrm{M}-\mathrm{m} 814 \mathrm{~W})$ colors, respectively. We thus recover the input value $(\Delta Y=0.082)$ with a good level of confidence.
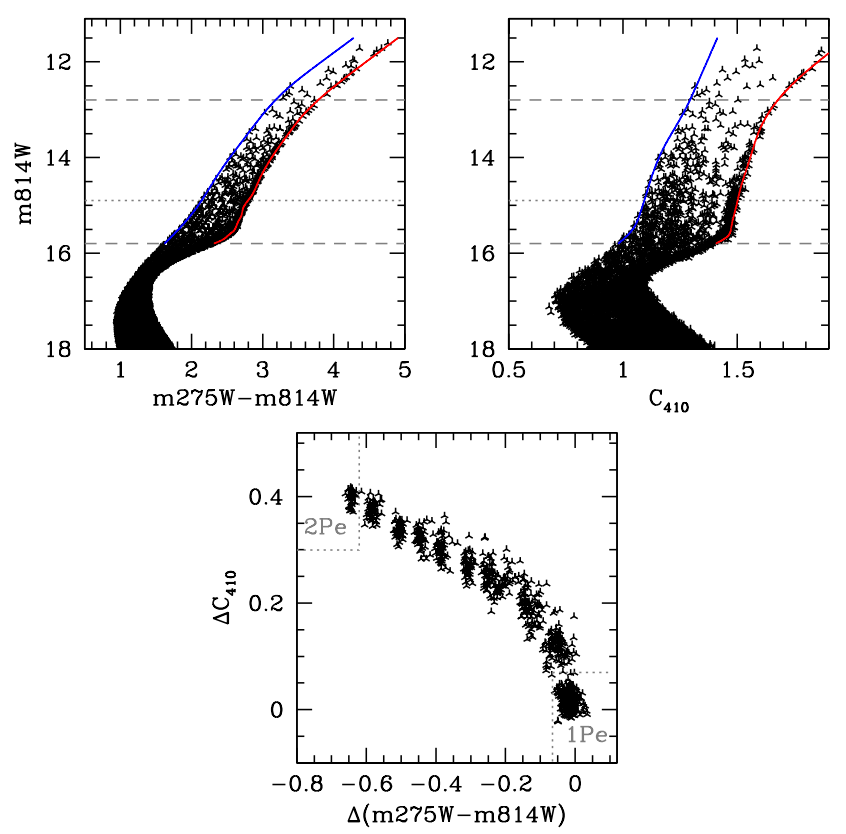

Fig. 12. Same as Fig. 7 but for a synthetic cluster with one-third stars with $Y=0.248$ and two-thirds stars with $Y=0.260$ to 0.600 with a flat distribution.

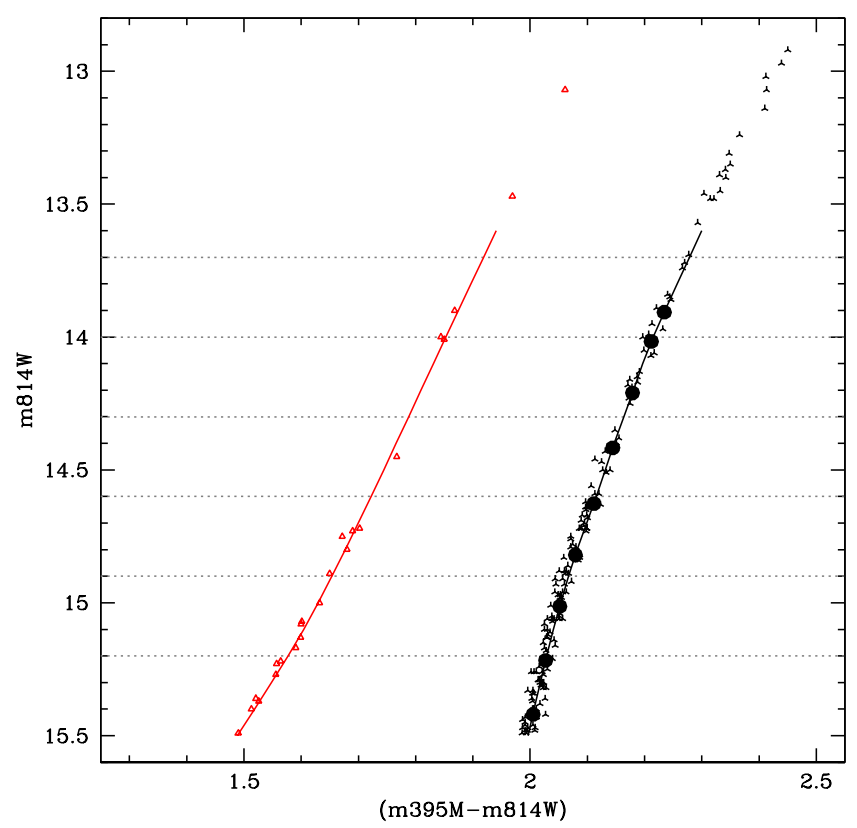

Fig. 13. Same as Fig. 8 but for a synthetic cluster with one-third stars with $Y=0.248$ and two-thirds stars with $Y=0.260$ to 0.600 with a flat distribution.

\subsubsection{Uniform distribution among second population}

We then run another test by building a synthetic cluster still made up of one-third 1P stars (3000 data points) drawn from the $Y=0.248$ isochrone, but with the remaining two-thirds of $2 \mathrm{P}$ stars (i.e., 6000 data points) spread uniformly between isochrones with $Y$ varying from 0.260 to 0.600 . For that, we create the isochrones characterized by $Y=0.430,0.470,0.500$, 0.530 , and 0.570 by linearly interpolating between the $Y=0.400$ and $Y=0.600$ isochrones. We create the five CMDs with these additional isochrones using the same method as described immediately above. We select 500 stars for each isochrone between 


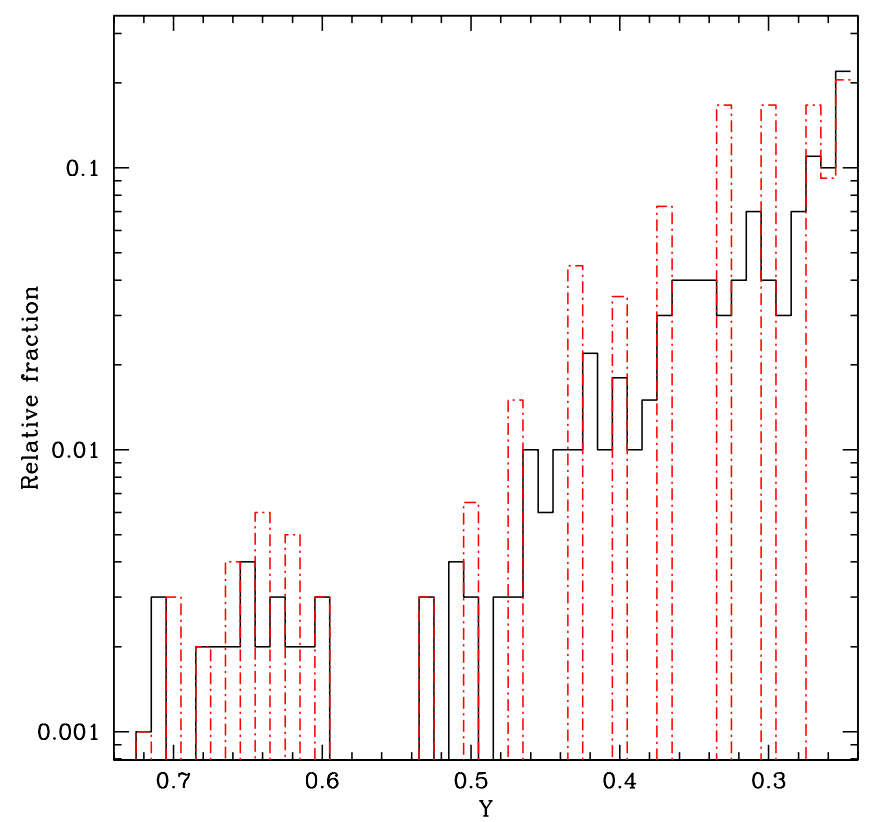

Fig. 14. Black solid histogram: Relative fraction of stars as a function of their helium content on the lower RGB in the models of Chantereau et al. (2016) and for an age of 13 Gyr. Red dashed histogram: Distribution we adopt to build synthetic clusters with populations as close as possible to those of Chantereau et al. (2016).

0.260 and 0.600 , which is $5.5 \%$ per isochrone, to obtain the final synthetic cluster. We then proceed as previously to determine $\Delta Y$. Figure 12 shows the definition of the fiducial lines and the chromosome map, together with the selected $1 \mathrm{Pe}$ and $2 \mathrm{Pe}$ stars. Figure 13 shows the $1 \mathrm{Pe}$ and $2 \mathrm{Pe}$ stars in the $\mathrm{m} 814 \mathrm{~W}$ versus $(\mathrm{m} 395 \mathrm{~N}-\mathrm{m} 814 \mathrm{~W})$ diagram. Here, we face a problem: the number of $2 \mathrm{Pe}$ stars is too small to apply the automatic process for defining the fiducial line of the $2 \mathrm{Pe}$ population. We therefore select the fiducial line by hand ${ }^{6}$, as done in the first step of the process that leads to the chromosome map. We then determine the $Y$ difference and we obtain $\Delta Y=0.362 \pm 0.005$. Using the other colors, $(\mathrm{m} 410 \mathrm{M}-\mathrm{m} 814 \mathrm{~W})$ and $(\mathrm{m} 467 \mathrm{M}-\mathrm{m} 814 \mathrm{~W})$, we obtain $\Delta Y=0.364 \pm 0.008$ and $\Delta Y=0.367 \pm 0.007$, respectively. The true $Y$ difference, being 0.352 , shows that we are able to measure it with good accuracy.

\subsubsection{Distribution of Chantereau et al.}

We finally move to clusters made of populations similar to those presented by Chantereau et al. (2016) within the framework of the FRMS scenario that fits the $\mathrm{O}-\mathrm{Na}$ anti-correlation in NGC 6752, and which is the most extreme case in terms of $\mathrm{He}$ enrichment. We use the distribution of stars according to their helium content shown in Fig. 6 of Chantereau et al. (2016) for an age of $13 \mathrm{Gyr}$ (at that age, their model predicts that $10 \%$ of the stars should have $Y>0.4$, from an initial fraction of $21 \%$ ). We adopt the distribution of the lower RGB as representative of the entire population of the cluster. Examination of Fig. 6 of Chantereau et al. (2016) reveals that this is a fair approximation for the MS up to the upper RGB. As we do not have synthetic photometry for the full distribution of $Y$ presented by Chantereau et al., we group bins of $Y$. This is illustrated in

\footnotetext{
6 We estimate the uncertainty on $\Delta Y$ resulting from the use of this manual process by performing ten repetitions on the same set of $1 \mathrm{Pe}$ and $2 \mathrm{Pe}$ populations. We find the uncertainty is of the order 0.004 .
}
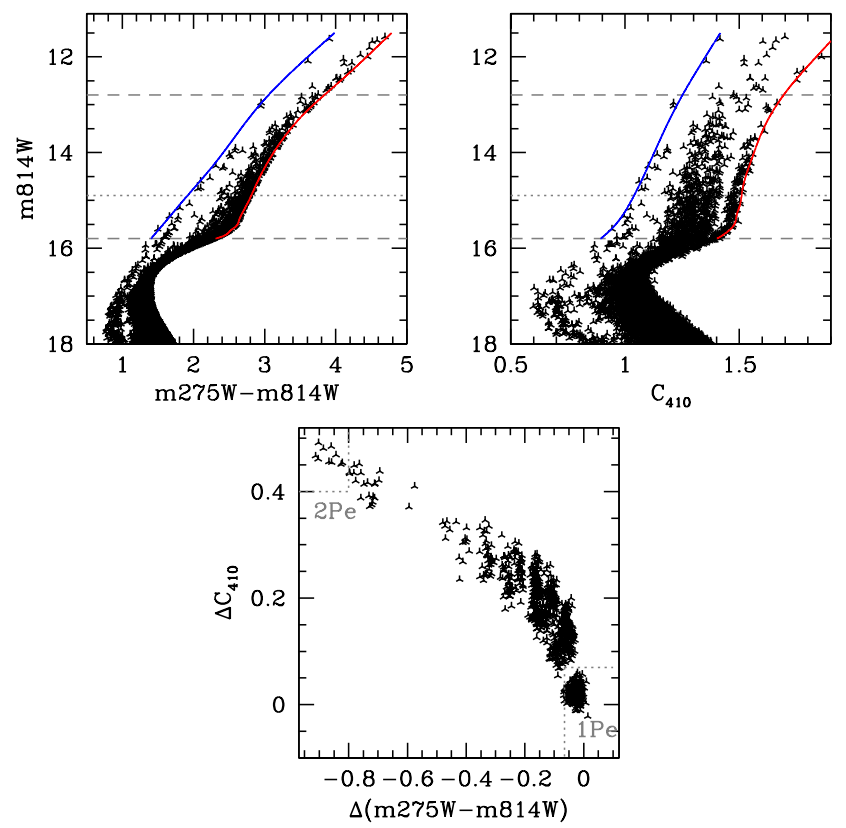

Fig. 15. Same as Fig. 7 but for a synthetic cluster with the populations of Chantereau et al. (2016).

Fig. 14. For instance, in the bin with $Y=0.30$ we gather the stars of Chantereau et al. with $Y=0.29,0.30$, and 0.31 . In addition, we linearly extrapolated our isochrones beyond $Y=0.6$ (up to $Y=0.72$ ) using our $Y=0.4$ and $Y=0.6$ synthetic isochrones. For the populations with $Y>0.6$ in Fig. 14 we group stars in bins of 0.2 in width (e.g., the $Y=0.64$ bin gathers stars with $Y=0.64$ and 0.65 from the distribution of Chantereau et al.). The most enriched population has $Y=0.72$ in our clusters, corresponding to $\Delta Y=0.472$. We built ten clusters.

We then perform the determination of the maximum helium mass fraction as in the previous example where a uniform distribution of stars with $Y>0.248$ was used. Figures 15 and 16 show the synthetic CMDs, chromosome map, and extreme populations in the $\mathrm{m} 814 \mathrm{~W}$ versus $(\mathrm{m} 395 \mathrm{M}-\mathrm{m} 814 \mathrm{~W}) \mathrm{CMD}$ in a representative example. We gather the results in Table 2 and Fig. 17. For some realizations of our synthetic clusters, and for some colors, we derive enrichments that are marginally compatible with the input value $\Delta Y=0.472$ : we obtain $\Delta Y=0.437 \pm 0.038$ for cluster 8 using the color $(\mathrm{m} 467 \mathrm{M}-\mathrm{m} 814 \mathrm{~W})$. However, the main result is that, on average, we determine a maximum $\Delta Y$ of about 0.43 , which is smaller than the input value of 0.472 . This is qualitatively understood by looking at Figs. 14 and 15. Stars with $Y>0.6$ have an almost flat distribution which translates into the extended population in upper left part of the chromosome map. When picking the stars identified as the $2 \mathrm{Pe}$ population, and to ensure a sufficient number of stars in that population, we include the stars with the highest $Y$, but also some stars with slightly smaller $Y$. We therefore tend to create a population with an average $Y$ that is smaller than 0.72 .

To further investigate this behavior, we show in Fig. 18 and Table 3 the effect of the selection of 2 Pe stars on the determination of the maximum helium difference. Choosing a larger population (population $2 \mathrm{Pe}(\mathrm{c})$ ) translates into a decrease in $\Delta Y$ by $\sim 0.03$, as expected because of the inclusion of stars with less extreme $Y$ in the $2 \mathrm{Pe}$ population. We also note that the values of $\Delta Y$ obtained with that selection of $2 \mathrm{Pe}$ stars are consistent with those obtained for other synthetic clusters reported in Table 2 and for which the $2 \mathrm{Pe}$ selection was more strict. Inversely, reducing 


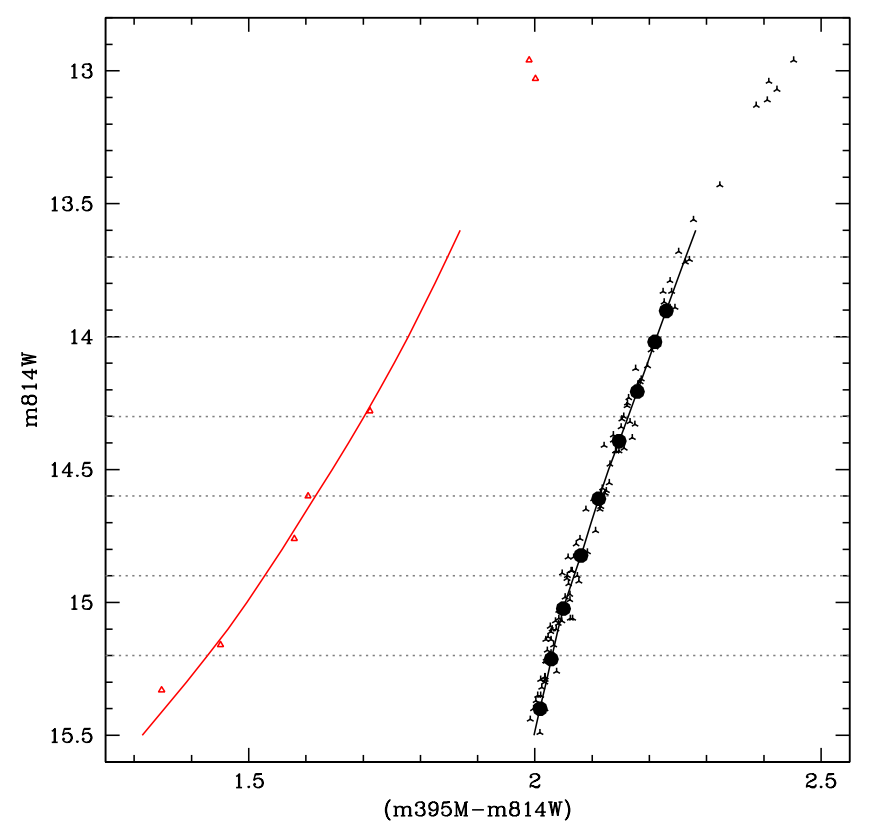

Fig. 16. Same as Fig. 8 but for a synthetic cluster with the populations of Chantereau et al. (2016).

Table 2. Helium difference recovered in ten synthetic clusters with a distribution of the population as in Fig. 14.

\begin{tabular}{lccc}
\hline \hline Cluster id & $\Delta Y_{395 N}$ & $\Delta Y_{410 M}$ & $\Delta Y_{467 M}$ \\
\hline 0 & $0.444 \pm 0.022$ & $0.448 \pm 0.014$ & $0.468 \pm 0.019$ \\
1 & $0.409 \pm 0.007$ & $0.411 \pm 0.006$ & $0.416 \pm 0.007$ \\
2 & $0.411 \pm 0.031$ & $0.416 \pm 0.021$ & $0.434 \pm 0.038$ \\
3 & $0.425 \pm 0.027$ & $0.434 \pm 0.027$ & $0.427 \pm 0.051$ \\
4 & $0.420 \pm 0.013$ & $0.429 \pm 0.008$ & $0.436 \pm 0.010$ \\
5 & $0.437 \pm 0.025$ & $0.438 \pm 0.023$ & $0.438 \pm 0.011$ \\
6 & $0.417 \pm 0.013$ & $0.421 \pm 0.009$ & $0.430 \pm 0.014$ \\
7 & $0.472 \pm 0.010$ & $0.472 \pm 0.017$ & $0.488 \pm 0.011$ \\
8 & $0.457 \pm 0.029$ & $0.460 \pm 0.020$ & $0.492 \pm 0.023$ \\
9 & $0.449 \pm 0.021$ & $0.460 \pm 0.023$ & $0.457 \pm 0.019$ \\
\hline Average & $0.430 \pm 0.004$ & $0.426 \pm 0.004$ & $0.441 \pm 0.004$ \\
\hline
\end{tabular}

Notes. $\Delta Y_{X}$ stands for the helium difference estimated from color $(X-$ $\mathrm{m} 814 \mathrm{~W}$ ) where $X$ is the magnitude in one of the filters $F 395 N, F 410 M$, or $F 467 M$.

the $2 \mathrm{Pe}$ population (2Pe(a) in Fig. 18), but still keeping a number of stars sufficient to define the 2Pe fiducial line, leads to a larger $\Delta Y$, which is consistent with the input value.

From these experiments, we conclude that for distributions of multiple populations with small numbers of stars with large chemical enrichments, such as that of Chantereau et al. (2016), the derived maximum helium enrichment critically depends on the selection of the $2 \mathrm{Pe}$ population. We also find that we slightly underestimate the maximum $Y$ of the cluster.

Chantereau et al. (2016) showed that the RGB contains less very enriched $(Y>0.6)$ stars than the MS (see their Fig. 5). We therefore expect that the determination of the maximum helium content of a GC using MS stars suffers less from the difficulties described above. To test this, we determined $\Delta Y$ as reported for RGB stars in Figs. 15 and 16 and Table 2 but focusing on MS stars. Figure 19 illustrates our selection of stars: those with m814 magnitudes between 16.9 and 18.1. We estimate the width of the $\mathrm{MS}$ at $\mathrm{m} 814=17.5$. The $1 \mathrm{Pe}$ and $2 \mathrm{Pe}$ stars are extracted from the

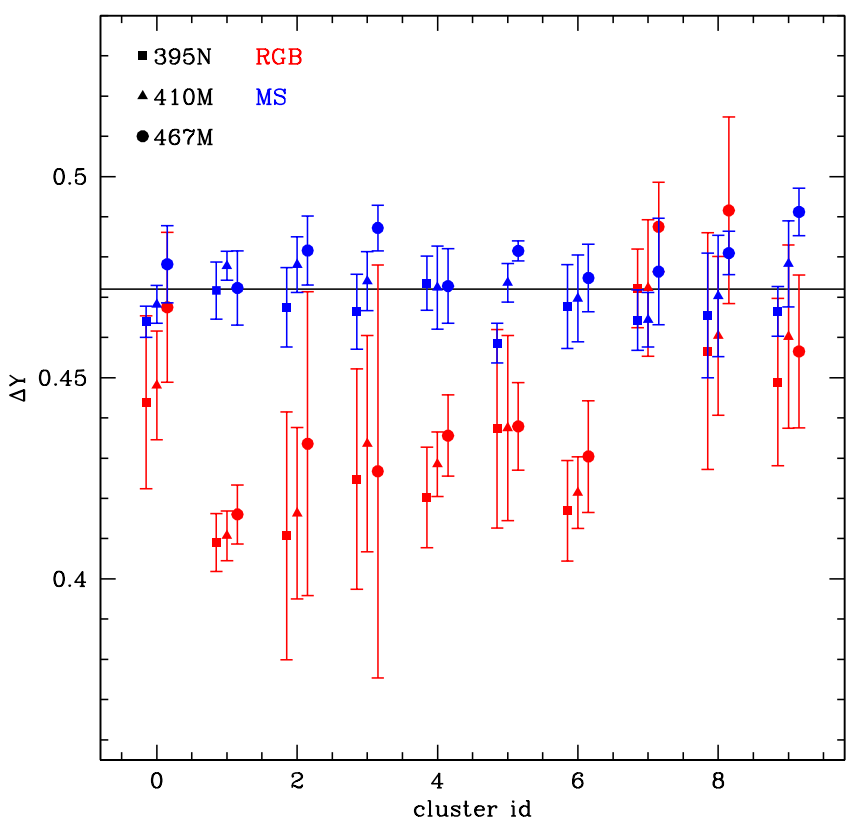

Fig. 17. Maximum helium difference $\Delta Y$ for ten synthetic clusters. Different symbols refer to different colors used for the determination. Blue (red) symbols stand for stars on the MS (RGB). The horizontal black solid line shows the true helium difference used to build the clusters.

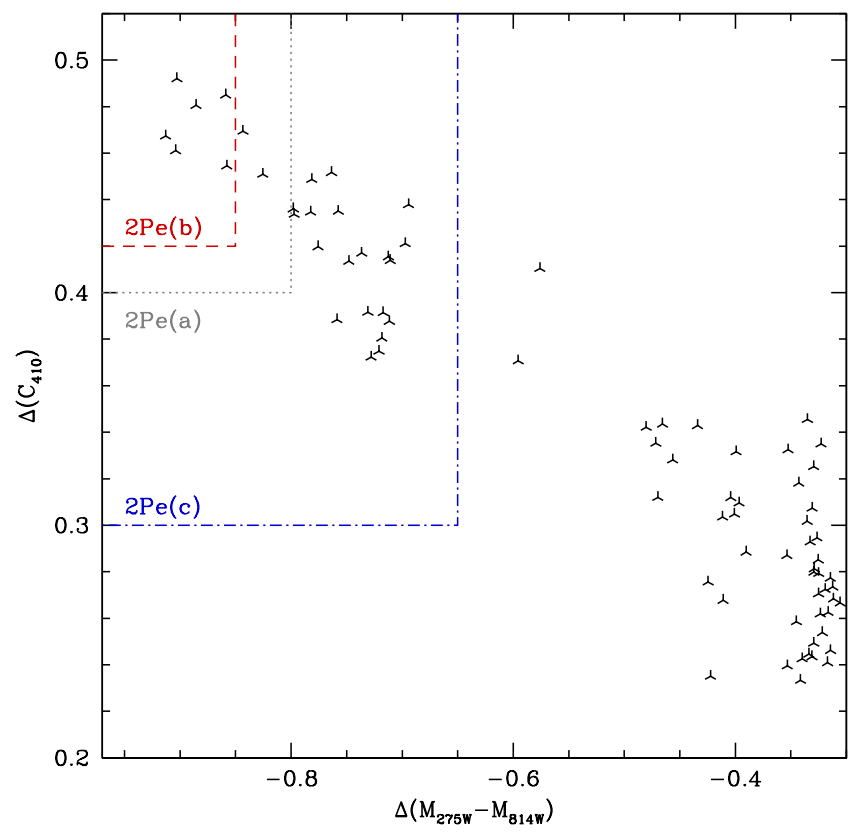

Fig. 18. Zoom into the upper left part of the chromosome map shown in the bottom panel of Fig. 15. The gray dotted lines mark the selection of $2 \mathrm{Pe}$ stars as in Fig. 15. The blue and red broken lines indicate alternative selections of $2 \mathrm{Pe}$ stars.

chromosome map as shown in the lower panel of Fig. 19. The results are gathered in Table 4 . Compared to Table 2 we see that the true helium mass fraction difference $(0.472)$ is indeed better recovered when using MS stars. A graphical representation of this result is given in Fig. 17 where we see that depending on the cluster simulation, $\Delta Y$ based on RGB stars may be underestimated, while for MS stars, the input value is always recovered. We thus conclude that for GCs that would have stellar populations similar to those of Chantereau et al. (2016), the study of the 
Table 3. Effect of the selection of 2Pe stars (see Fig. 18) on the determination of the maximum helium difference in synthetic cluster number 0 .

\begin{tabular}{lccc}
\hline \hline 2Pe selection & $\Delta Y_{395 N}$ & $\Delta Y_{410 M}$ & $\Delta Y_{467 M}$ \\
\hline 2Pe(a) & $0.444 \pm 0.022$ & $0.448 \pm 0.014$ & $0.468 \pm 0.019$ \\
$2 \mathrm{Pe}(\mathrm{b})$ & $0.458 \pm 0.018$ & $0.471 \pm 0.011$ & $0.489 \pm 0.006$ \\
$2 \mathrm{Pe}(\mathrm{c})$ & $0.410 \pm 0.040$ & $0.410 \pm 0.034$ & $0.418 \pm 0.032$ \\
\hline
\end{tabular}

Notes. The first column gives the label of the $2 \mathrm{Pe}$ selection. The following columns give the helium difference estimated using the same three colors as in Table 2.
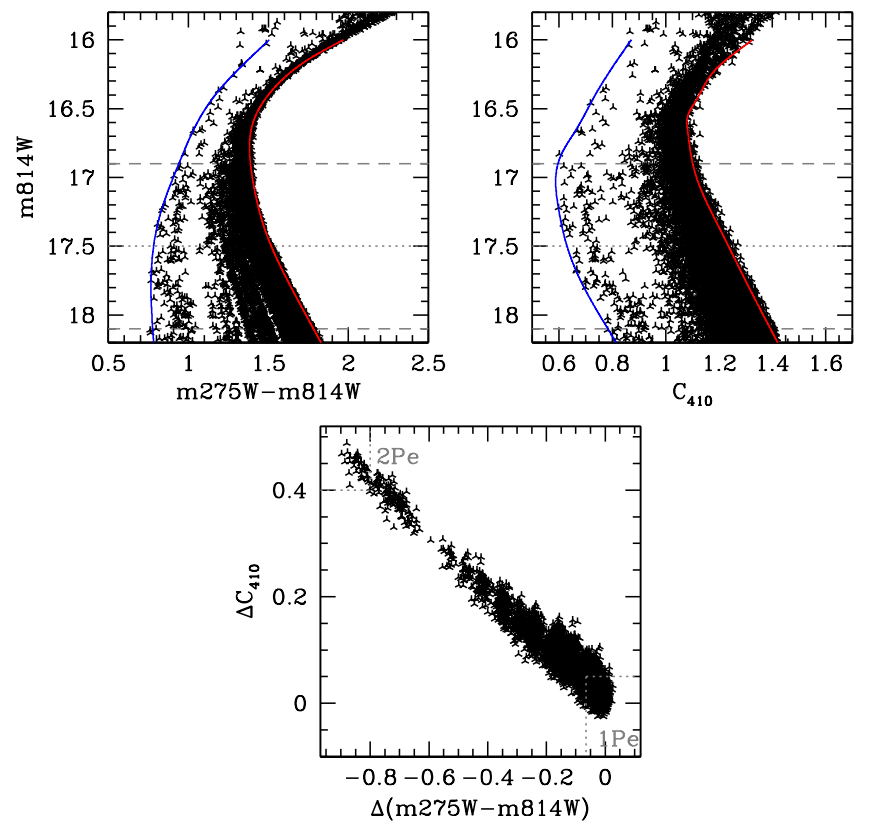

Fig. 19. Same as Fig. 15 but for MS stars.

Table 4. Same as Table 2 but for MS stars.

\begin{tabular}{lccc}
\hline \hline Cluster id & $\Delta Y_{395 N}$ & $\Delta Y_{410 M}$ & $\Delta Y_{467 M}$ \\
\hline 0 & $0.464 \pm 0.004$ & $0.468 \pm 0.005$ & $0.478 \pm 0.010$ \\
1 & $0.472 \pm 0.007$ & $0.478 \pm 0.004$ & $0.472 \pm 0.009$ \\
2 & $0.468 \pm 0.010$ & $0.478 \pm 0.007$ & $0.482 \pm 0.009$ \\
3 & $0.466 \pm 0.009$ & $0.474 \pm 0.007$ & $0.487 \pm 0.006$ \\
4 & $0.473 \pm 0.007$ & $0.472 \pm 0.010$ & $0.473 \pm 0.009$ \\
5 & $0.459 \pm 0.005$ & $0.474 \pm 0.005$ & $0.482 \pm 0.003$ \\
6 & $0.468 \pm 0.010$ & $0.470 \pm 0.011$ & $0.475 \pm 0.008$ \\
7 & $0.464 \pm 0.008$ & $0.464 \pm 0.007$ & $0.476 \pm 0.013$ \\
8 & $0.466 \pm 0.016$ & $0.470 \pm 0.015$ & $0.481 \pm 0.005$ \\
9 & $0.467 \pm 0.006$ & $0.478 \pm 0.011$ & $0.491 \pm 0.006$ \\
\hline Average & $0.465 \pm 0.002$ & $0.473 \pm 0.002$ & $0.482 \pm 0.002$ \\
\hline
\end{tabular}

maximum helium content should be preferentially performed on MS stars, provided sufficient data quality.

\subsubsection{Steep distribution}

In this section we try to answer the question of whether or not we can miss very helium-rich stars if multiple populations follow a steeper distribution than that presented by Chantereau et al. (2016). To this end, we define an artificial distribution as shown in Fig. 20. We start from the distribution of Chantereau et al.

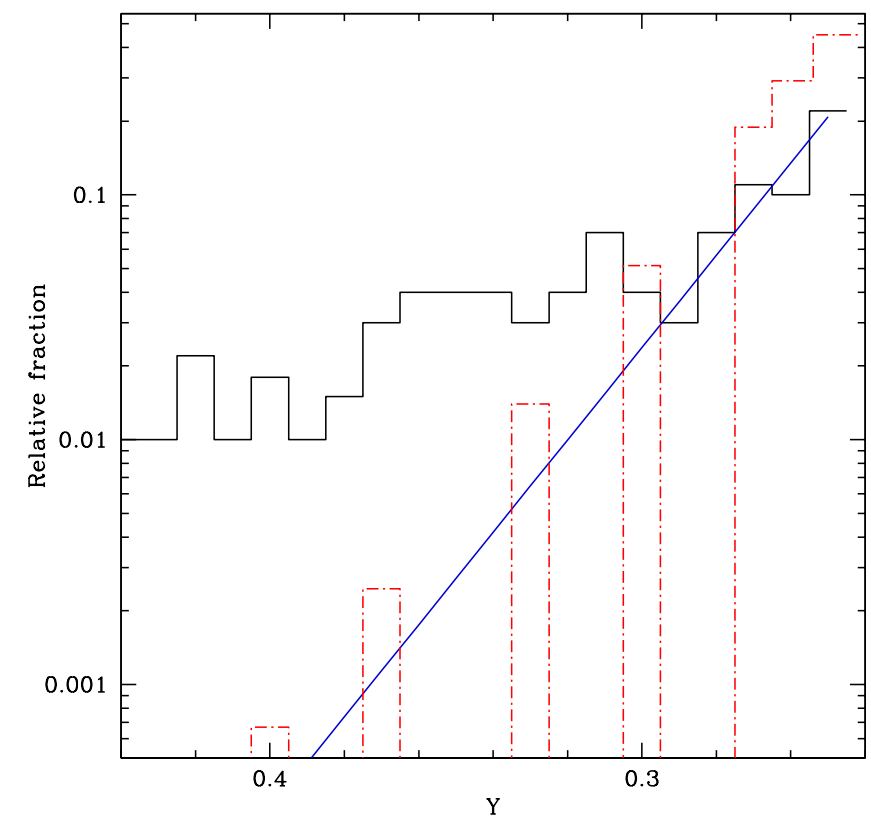

Fig. 20. Black solid histogram: Relative fraction of stars as a function of their helium content on the lower RGB in the models of Chantereau et al. (2016) and for an age of 13 Gyr. Blue solid line: Linear interpolation of Chantereau's distribution between $Y=0.248$ and 0.290 , and extrapolation up to $Y=0.400$. Red dashed histogram: Adopted distribution based on the previous interpolation and re-scaling so that the sum of relative fractions is equal to 1 .

(2016) between $Y=0.248$ and $Y=0.29$. We linearly interpolate their distribution in this range of $Y$, and then extrapolate the resulting distribution up to $Y=0.400$ (blue line in Fig. 20). We thus obtain a much steeper distribution of populations as a function of $Y$ than that of Chantereau et al. for $Y>0.29$. According to Milone et al. (2018) and Sect. 3.1, the maximum helium mass fraction observed in NGC 6752 is about 0.29 . Our artificial distribution can therefore be seen as a test case where only a few stars with $Y>0.29$ are present in synthetic clusters. We then try to see if they are recovered or missed when determining $\Delta Y$.

We built ten synthetic clusters using the above distribution. We use only $Y$ values for which isochrones are available, that is, $Y=0.248,0.260,0.270,0.300,0.330,0.370$, and 0.400 (red bins in Fig. 20). We perform the $\Delta Y$ determination as in the previous sections. The results are gathered in Fig. 21. Using RGB stars, we are not able to recover the input value $(\Delta Y=0.152)$, but we can still detect stars with $Y>0.29$ (i.e., $\Delta Y=0.042$ ). If present, and under the assumption that their distribution follows that described above, stars more helium-rich than $Y=0.29$ should therefore be detectable. On the MS, we confirm the trend seen in Sect. 3.2.3 that higher values of $\Delta Y$ are recovered. However, with the present distribution, the maximum helium mass fraction of the cluster is also missed on the MS due to the very small number of highly enriched stars. This is different from Sect. 3.2.3 where on the MS the initial value of $\Delta Y$ could be retrieved.

\section{Discussion}

Our method for determining the maximum helium mass fraction in GCs is slightly less automated than that presented by Milone et al. (2018). In particular, the selection of the extreme $1 \mathrm{Pe}$ and $2 \mathrm{Pe}$ populations is made visually in our case, while it relies on a more sophisticated treatment in Milone et al. (2018). 


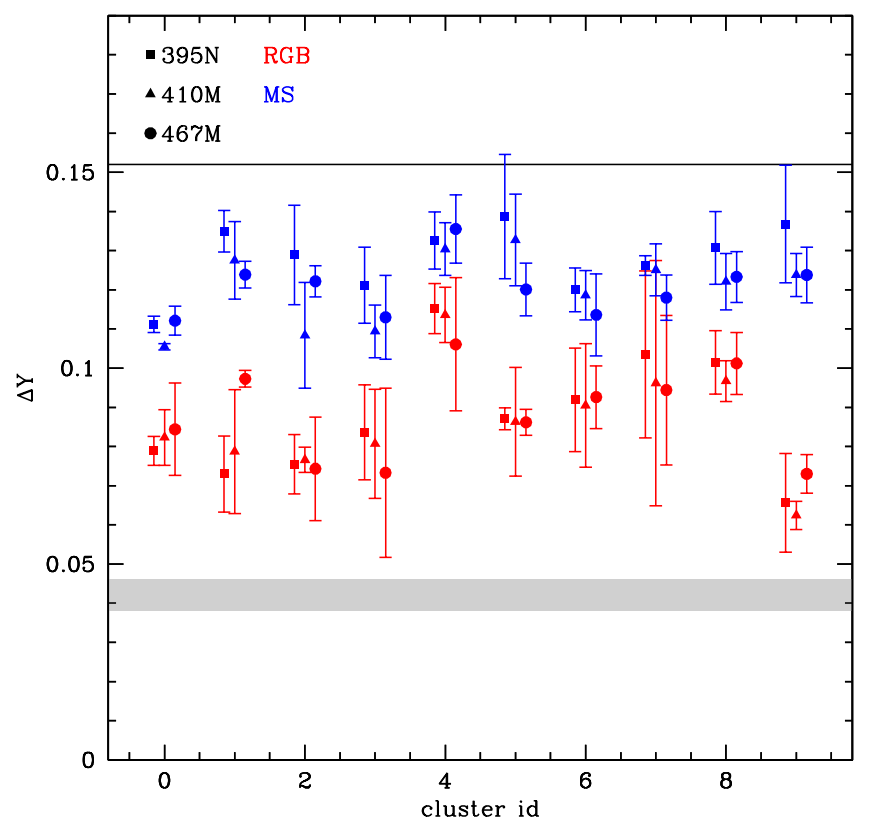

Fig. 21. Same as Fig. 17 but for the distribution of the population described in Sect. 3.2.4. The gray area marks the observed value of $\Delta Y$ in NGC 6752.

We tested the effect of the 2Pe selection in Sect. 3.2.3 and Fig. 18, and argue that the choice of $2 \mathrm{Pe}$ stars can affect the value of the maximum $Y$. However, qualitatively, stars much more He-rich than the observed limit of 0.042 are easily detected regardless of the $2 \mathrm{Pe}$ selection. In addition, the automated method of Milone et al. (2018) works well when 2Pe stars are clearly identified by an overdensity in the chromosome map, but there are clusters for which no such overdensity is visible. For instance, the chromosome map of NGC 6752 itself, presented in the bottom panel of Fig. 7, shows a rather uniform distribution along the $2 \mathrm{P}$ sequence (i.e., from the bottom right corner up to the upper left corner). Hence, we argue that the choice of $2 \mathrm{Pe}$ stars in our study is not drastically different from Milone et al. (2018).

He-rich populations may be contaminated by blue stragglers and their descendants which would populate similar regions of CMDs. Marino et al. (2019) showed that candidate evolved blue stragglers should be located preferentially along the $1 \mathrm{P}$ sequence, and thus would not contaminate $2 \mathrm{Pe}$ stars. However, one may wonder whether blue stragglers resulting from the merger of two $2 \mathrm{P}$ stars could produce stars along the $2 \mathrm{P}$ sequence too. This needs to be investigated further. Binaries can also contaminate the 2P sequence. As shown by Martins et al. (2020), the presence of a companion among RGB stars will tend to displace a star up and left in the chromosome map. The magnitude of the displacement depends on the relative brightness and effective temperature of the two stars. Hence, stars along the $2 \mathrm{G}$ sequence, with medium $Y$ but with a companion, may be moved to the position of single stars with higher $Y$. Consequently, the extremity of the $2 \mathrm{P}$ sequence may contain stars with less extreme $Y$. This could lead to overestimation of the maximum $Y$. The binary fraction among GCs is low, usually below $10 \%$ (Sollima et al. 2007; Milone et al. 2012; Ji \& Bregman 2015), and their effect on the maximum $Y$ should therefore be limited; unless the number of very He-rich stars is also small, as in the distribution of Chantereau et al. (2016). In any case, if binaries are present, the maximum $Y$ determined from photometry is probably overestimated.
With these limitations in mind, and given the results of the present study, it is unlikely that NGC 6752 contains stars with $Y \gtrsim 0.3$. If multiple populations were formed as predicted by the FRMS scenario developed by Decressin et al. (2007a) and Chantereau et al. (2015, 2016), a wide distribution of $Y$, from 0.248 to 0.72 , should be present among stars either on the MS or the RGB, with $39 \%$ of the stars with $Y>0.3$ and $10 \%$ with $Y>0.4$ at 13 Gyr. Our study reveals that while we may not retrieve the most helium-rich objects on the RGB, we should still be able to detect stars with $Y$ as high as 0.65 (see Fig. 17). This is clearly in contrast with results based on HST photometry indicating $Y$ no higher than 0.3 . We have shown that even in the case of a $Y$ distribution much steeper than that predicted by Chantereau et al. (2016) we would be able to find stars with helium enrichment beyond the observed value.

A prediction of our study dedicated to NGC 6752 is that the maximum helium content of GC stars, if it follows a distribution where helium-rich stars are less numerous than heliumpoor ones, is best determined on the MS rather than on the RGB. This is due to the faster evolution of He-rich stars and consequently the larger number of stars on the MS than on the RGB (Demarque \& Mengel 1973; D'Antona et al. 2010; Chantereau et al. 2016). However, the maximum $Y$ we obtain on the MS using HST data of NGC 6752 does not indicate a significant difference compared to the value found on the RGB.

All in all, the present results strongly suggest that stars in NGC 6752 do not follow the distribution predicted by the FRMS model presented in Chantereau et al. (2016), and consequently, multiple populations were not likely formed out of material polluted by this type of object. This conclusion applies only to NGC 6752, and additional studies of clusters with different ages, metallicities, and masses are required to see whether generalizations can be made. A wider study of this kind is necessary to investigate whether or not the current observational limit of $Y \sim 0.4$ is robust and to investigate whether or not it suffers from observational limitations. As recalled in Sect. 1, this limit is a key prediction of some models, but also a building block of some others. In particular, the formation of multiple populations caused by pollution of material ejected from a super-massive star (SMS) originally assumes that the SMS stops its evolution when its core helium content reaches 0.4 , and is further dislocated by instabilities and/or strong stellar winds (Denissenkov \& Hartwick 2014). This assumption was dictated by the observational fact that $Y$ appears to be no higher than 0.4 in the most extreme GC populations. On the other hand, hot-hydrogen burning products with low helium abundances in agreement with the photometric constraints can be ejected by SMSs in the case where they are continuously rejuvenated by runaway stellar collisions (Gieles et al. 2018).

Pollution by material produced in massive AGB stars predicts that the helium distribution among multiple populations reaches a maximum of $\sim 0.38$ (e.g., D'Ercole et al. 2010). This is consistent with the current observational limit, and would make these objects good polluter candidates if other problems were not linked to their use, as summarized in Renzini et al. (2015) and Bastian \& Lardo (2018) for instance. Other important factors are the time constraints on the dilution by pristine material, as also highlighted by (D'Ercole et al. 2011, see also D'Ercole et al. 2016), and the mass budget issue (e.g., Prantzos \& Charbonnel 2006; Krause et al. 2016). The transformation of the $\mathrm{Na}-\mathrm{O}$ correlation in AGB ejecta into a $\mathrm{Na}-\mathrm{O}$ anticorrelation, as seen in all GCs, also requires specific conditions for mixing of pristine material with nucleosynthesis products (e.g., Ventura \& D’Antona 2008; Karakas 2010). In addition, the 
$\mathrm{C}+\mathrm{N}+\mathrm{O}$ sum of AGB ejecta is not conserved (Decressin et al 2009), contrary to what is deduced from spectroscopic analysis of stars in NGC 6752 (Yong et al. 2015).

The role of massive binaries in the multiple population phenomenon remains to be investigated. de Mink et al. (2009) showed that these objects could potentially produce the required abundance patterns. But their study is limited to a $20+15 M_{\odot}$ system on a 12-day orbit and in which both components have reached synchronization. The average helium mass fraction of the ejecta of this binary system is 0.3 . Material ejected at later phases of the system's evolution has $Y$ as large as $\sim 0.63$ (see Fig. 1 of de Mink et al. 2009). The average helium content is therefore consistent with the current maximum $Y$ observed in GCs. However binary evolution depends not only on the properties and evolution of the components, but also on the parameters of the system (separation, eccentricity, mass ratio, mass transfer efficiency); see for example Menon et al. (2020). Additionally, the maximum central temperature of the considered mass domain does not reach the high values required to build the Mg-Al anti-correlation (e.g., Prantzos et al. 2007, 2017). A wider study involving population synthesis is therefore required to assess the impact of massive binaries on the origin of multiple populations in GCs.

\section{Conclusion}

In this study, we investigated the determination of the maximum helium mass fraction in stars of the GC NGC 6752. Our goal was to decipher whether we really detect the most He-rich stars with present-day photometric methods, or miss them. We relied on the work of Chantereau et al. (2016) who produced isochrones with various chemical compositions corresponding to different degrees of pollution by FRMS which is an extreme case in terms of He enrichment. We computed synthetic spectra along these isochrones using the atmosphere code ATLAS 12 and the spectral synthesis code SYNTHE. The resulting spectra were used to compute synthetic photometry in the following HST filters: WFC3 $F 275 W$, WFC3 $F 336 W$, WFC3 $F 395 N$, WFC3 $F 410 M$, WFC3 $F 467 M$, ACS $F 606 W$, and ACS $F 814 W$. We compared the synthetic colors with data of NGC 6752 obtained by Milone et al. (2013). The different CMDs are usually reasonably well reproduced, although offsets exist between synthetic and observed sequences (MS, TO, RGB).

We re-determined the maximum helium mass fraction of stars in NGC 6752 using a method very similar to that of Milone et al. (2018). Our results are consistent with those of Milone at al. We built synthetic clusters with various populations characterized by their He content (they also have different composition in light elements). We validated our method on simple population distributions, ensuring that we are able to recover the input maximum $Y$. We subsequently created synthetic clusters following the distribution presented by Chantereau et al (2016), that is, with stars with $Y$ of between 0.248 and 0.72 . We show that on the RGB, we slightly underestimate the maximum $Y$, but by a relatively small amount $(\sim 0.05)$. On the MS, we retrieve the input value. In any case, even on the RGB the maximum $Y$ we determine in synthetic clusters is higher than the observed value of $0.042 \pm 0.004$ (Milone et al. 2018). We tested that even if populations followed a steeper $Y$ distribution than that of Chantereau et al. (2016), stars with $Y$ higher than 0.042 are recovered (in that case the maximum $Y$ is underestimated both on the RGB and MS). Finally, we determined the maximum $Y$ on the MS of NGC 6752 using HST data and find it to be consistent with the value obtained on the RGB.
These results indicate that multiple populations in NGC 6752 have a $Y$ distribution that is very likely not that assumed by Chantereau et al. (2016) in the framework of the FRMS scenario. Our results also show that in the specific case studied here, the maximum helium mass fraction determined from observations is probably the true value (i.e., there are no stars more He-rich in that cluster). We stress that although we have focused on the specific case of pollution by FRMS, our results apply to any scenario that would predict a strong He enrichment among $2 \mathrm{P}$ stars (Salaris et al. 2006; Pietrinferni et al. 2009; Cassisi et al. 2013; Dotter et al. 2015). Our results need to be extended to other clusters spanning a range of parameters (age, metallicity, mass) in order to confirm that FRMSs are not responsible for the multiple populations in GCs and to better constrain the scenario that produce them.

Acknowledgements. We thank an anonymous referee for a positive report. We thank A. Milone for sharing HST photometry of NGC 6752 and for interesting discussions. This work was supported by the Swiss National Science Foundation (Project 200020-192039 PI C.C.). This work was supported by the Agence Nationale de la Recherche grant POPSYCLE number ANR-19-CE31-0022.

\section{References}

Ali, S. S., Bremer, M. N., Phillipps, S., \& De Propris, R. 2018, MNRAS, 480, 2236

Bastian, N., \& Lardo, C. 2018, ARA\&A, 56, 83

Bedin, L. R., Piotto, G., Anderson, J., et al. 2004, ApJ, 605, L125

Bohlin, R. C., Gordon, K. D., \& Tremblay, P. E. 2014, PASP, 126, 711

Bowman, W. P., Pilachowski, C. A., van Zee, L., et al. 2017, AJ, 154, 131

Briley, M. M., Bell, R. A., Hoban, S., \& Dickens, R. J. 1990, ApJ, 359, 307

Caloi, V., \& D'Antona, F. 2005, A\&A, 435, 987

Campbell, S. W., D’Orazi, V., Yong, D., et al. 2013, Nature, 498, 198

Cardelli, J. A., Clayton, G. C., \& Mathis, J. S. 1989, ApJ, 345, 245

Carretta, E. 2015, ApJ, 810, 148

Carretta, E. 2019, A\&A, 624, A24

Cassisi, S., \& Salaris, M. 2020, A\&ARv, 28, 5

Cassisi, S., Mucciarelli, A., Pietrinferni, A., Salaris, M., \& Ferguson, J. 2013, A\&A, 554, A19

Chantereau, W., Charbonnel, C., \& Decressin, T. 2015, A\&A, 578, A117

Chantereau, W., Charbonnel, C., \& Meynet, G. 2016, A\&A, 592, A111

Chantereau, W., Salaris, M., Bastian, N., \& Martocchia, S. 2019, MNRAS, 484, 5236

Charbonnel, C., \& Zahn, J. P. 2007, A\&A, 467, L15

Coelho, P. R. T. 2014, MNRAS, 440, 1027

Cohen, J. G. 1978, ApJ, 223, 487

D'Antona, F., \& Caloi, V. 2004, ApJ, 611, 871

D’Antona, F., Bellazzini, M., Caloi, V., et al. 2005, ApJ, 631, 868

D'Antona, F., Caloi, V., \& Ventura, P. 2010, MNRAS, 405, 2295

de Mink, S. E., Pols, O. R., Langer, N., \& Izzard, R. G. 2009, A\&A, 507, L1

Decressin, T., Meynet, G., Charbonnel, C., Prantzos, N., \& Ekström, S. 2007a, A\&A, 464, 1029

Decressin, T., Charbonnel, C., \& Meynet, G. 2007b, A\&A, 475, 859

Decressin, T., Charbonnel, C., Siess, L., et al. 2009, A\&A, 505, 727

Demarque, P., \& Mengel, J. G. 1973, A\&A, 22, 121

Denisenkov, P. A., \& Denisenkova, S. N. 1990, Sov. Astron. Lett., 16, 275

Denissenkov, P. A., \& Hartwick, F. D. A. 2014, MNRAS, 437, L21

Denissenkov, P. A., VandenBerg, D. A., Kopacki, G., \& Ferguson, J. W. 2017, ApJ, 849, 159

D’Ercole, A., D'Antona, F., Ventura, P., Vesperini, E., \& McMillan, S. L. W. 2010, MNRAS, 407, 854

D'Ercole, A., D'Antona, F., \& Vesperini, E. 2011, MNRAS, 415, 1304

D'Ercole, A., D'Antona, F., \& Vesperini, E. 2016, MNRAS, 461, 4088

di Criscienzo, M., Ventura, P., D’Antona, F., Milone, A., \& Piotto, G. 2010, MNRAS, 408, 999

Doherty, C. L., Gil-Pons, P., Lau, H. H. B., Lattanzio, J. C., \& Siess, L. 2014, MNRAS, 437, 195

Dotter, A., Ferguson, J. W., Conroy, C., et al. 2015, MNRAS, 446, 1641

Dupree, A. K., \& Avrett, E. H. 2013, ApJ, 773, L28

Elmegreen, B. G. 2017, ApJ, 836, 80

Ferraro, F. R., Paltrinieri, B., Fusi Pecci, F., et al. 1997, ApJ, 484, L145

Gaia Collaboration (Helmi, A., et al.) 2018, A\&A, 616, A12

Gieles, M., Charbonnel, C., Krause, M. G. H., et al. 2018, MNRAS, 478, 2461 
Gratton, R. G., Bragaglia, A., Carretta, E., et al. 2003, A\&A, 408, 529 Gratton, R. G., Bragaglia, A., Carretta, E., et al. 2005, A\&A, 442, 947 Gratton, R. G., Lucatello, S., Bragaglia, A., et al. 2007, A\&A, 464, 953 Gratton, R., Bragaglia, A., Carretta, E., et al. 2019, A\&ARv, 27, 8

Greggio, L., \& Renzini, A. 1990, ApJ, 364, 35

Harris, W. E. 1996, AJ, 112, 1487

Henkel, K., Karakas, A. I., \& Lattanzio, J. C. 2017, MNRAS, 469, 4600

Iben, I., Jr., \& Faulkner, J. 1968, ApJ, 153, 101

Izzard, R. G., de Mink, S. E., Pols, O. R., et al. 2013, Mem. Soc. Astron. It., 84 171

Ji, J., \& Bregman, J. N. 2015, ApJ, 807, 32

Johnson, C. I., Caldwell, N., Rich, R. M., Pilachowski, C. A., \& Hsyu, T. 2016, AJ, 152, 21

Karakas, A. I. 2010, in Star Clusters: Basic Galactic Building Blocks Throughout Time and Space, eds. R. de Grijs, \& J. R. D. Lépine, 266, 161

King, I. R., Bedin, L. R., Cassisi, S., et al. 2012, AJ, 144, 5

Kraft, R. P. 1994, PASP, 106, 553

Krause, M. G. H., Charbonnel, C., Bastian, N., \& Diehl, R. 2016, A\&A, 587, A53

Kudryashov, A. D., \& Tutukov, A. V. 1988, Astronomicheskij Tsirkulyar, 1525,

Kurucz, R. L. 2005, Mem. Soc. Astron. It. Suppl., 8, 76

Kurucz, R. L. 2014, in Model Atmosphere Codes: ATLAS12 and ATLAS9, eds. E. Niemczura, B. Smalley, \& W. Pych, 39

Lardo, C., Pancino, E., Mucciarelli, A., \& Milone, A. P. 2012, A\&A, 548, A107

Lee, Y.-W., Joo, S.-J., Han, S.-I., et al. 2005, ApJ, 621, L57

Lind, K., Primas, F., Charbonnel, C., Grundahl, F., \& Asplund, M. 2009, A\&A, 503, 545

Marcolini, A., Gibson, B. K., Karakas, A. I., \& Sánchez-Blázquez, P. 2009, MNRAS, 395, 719

Marino, A. F., Milone, A. P., Przybilla, N., et al. 2014, MNRAS, 437, 1609

Marino, A. F., Milone, A. P., Sills, A., et al. 2019, ApJ, 887, 91

Martins, F., Morin, J., Charbonnel, C., Lardo, C., \& Chantereau, W. 2020, A\&A, 635, A52

Masseron, T., García-Hernández, D. A., Mészáros, S., et al. 2019, A\&A, 622, A191

Menon, A., de Mink, S. E., Langer, N., et al. 2020, MNRAS, submitted [arXiv:2011.13459]

Mészáros, S., Martell, S. L., Shetrone, M., et al. 2015, AJ, 149, 153

Meynet, G., Decressin, T., \& Charbonnel, C. 2008, Mem. Soc. Astron. It., 79, 584

Michaud, G., Richer, J., \& Richard, O. 2008, ApJ, 675, 1223

Milone, A. P. 2015, MNRAS, 446, 1672
Milone, A. P., Piotto, G., Bedin, L. R., et al. 2012, A\&A, 540, A16 Milone, A. P., Marino, A. F., Piotto, G., et al. 2013, ApJ, 767, 120 Milone, A. P., Piotto, G., Renzini, A., et al. 2017, MNRAS, 464, 3636 Milone, A. P., Marino, A. F., Renzini, A., et al. 2018, MNRAS, 481, 5098 Milone, A. P., Marino, A. F., Renzini, A., et al. 2020, MNRAS, 497, 3846 Nardiello, D., Libralato, M., Piotto, G., et al. 2018, MNRAS, 481, 3382 Norris, J., Cottrell, P. L., Freeman, K. C., \& Da Costa, G. S. 1981, ApJ, 244, 205 Pancino, E., Romano, D., Tang, B., et al. 2017, A\&A, 601, A112

Pasquini, L., Mauas, P., Käufl, H. U., \& Cacciari, C. 2011, A\&A, 531, A35 Peterson, R. C. 1980, ApJ, 237, L87

Pietrinferni, A., Cassisi, S., Salaris, M., Percival, S., \& Ferguson, J. W. 2009, ApJ, 697, 275

Piotto, G., Bedin, L. R., Anderson, J., et al. 2007, ApJ, 661, L53

Piotto, G., Milone, A. P., Bedin, L. R., et al. 2015, AJ, 149, 91 Prantzos, N., \& Charbonnel, C. 2006, A\&A, 458, 135

Prantzos, N., Charbonnel, C., \& Iliadis, C. 2007, A\&A, 470, 179 Prantzos, N., Charbonnel, C., \& Iliadis, C. 2017, A\&A, 608, A28 Quievy, D., Charbonneau, P., Michaud, G., \& Richer, J. 2009, A\&A, 500, 1163

Renzini, A., Bragaglia, A., Ferraro, F. R., et al. 1996, ApJ, 465, L23

Renzini, A., D’Antona, F., Cassisi, S., et al. 2015, MNRAS, 454, 4197

Rood, R. T. 1973, ApJ, 184, 815

Salaris, M., Weiss, A., Ferguson, J. W., \& Fusilier, D. J. 2006, ApJ, 645, 1131

Sbordone, L., Salaris, M., Weiss, A., \& Cassisi, S. 2011, A\&A, 534, A9

Schlegel, D. J., Finkbeiner, D. P., \& Davis, M. 1998, ApJ, 500, 525

Shetrone, M. D. 2003, ApJ, 585, L45

Sneden, C., Kraft, R. P., Prosser, C. F., \& Langer, G. E. 1992, AJ, 104, 2121

Sollima, A., Beccari, G., Ferraro, F. R., Fusi Pecci, F., \& Sarajedini, A. 2007, MNRAS, 380, 781

Szécsi, D., \& Wünsch, R. 2019, ApJ, 871, 20

Tailo, M., Di Criscienzo, M., D’Antona, F., Caloi, V., \& Ventura, P. 2016, MNRAS, 457, 4525

Thévenin, F., Charbonnel, C., de Freitas Pacheco, J. A., et al. 2001, A\&A, 373, 905

Valcarce, A. A. R., Catelan, M., Alonso-García, J., Contreras Ramos, R., \& Alves, S. 2016, A\&A, 589, A126

VandenBerg, D. A., \& Denissenkov, P. A. 2018, ApJ, 862, 72

Ventura, P., \& D'Antona, F. 2008, MNRAS, 385, 2034

Ventura, P., \& D'Antona, F. 2009, A\&A, 499, 835

Ventura, P., D'Antona, F., Mazzitelli, I., \& Gratton, R. 2001, ApJ, 550, L65

Villanova, S., Geisler, D., Piotto, G., \& Gratton, R. G. 2012, ApJ, 748, 62

Wang, Y., Primas, F., Charbonnel, C., et al. 2016, A\&A, 592, A66

Wang, Y., Primas, F., Charbonnel, C., et al. 2017, A\&A, 607, A135

Yong, D., Grundahl, F., \& Norris, J. E. 2015, MNRAS, 446, 3319 
Appendix A: Chemical composition adopted for multiple populations

Table A.1 gives the chemical composition of the different mixtures used to compute evolutionary tracks and isochrones according to Chantereau et al. (2015) adapted for
$[\mathrm{Fe} / \mathrm{H}]=-1.53$. Each mixture is labeled according to its helium mass fraction $Y$. The $Y=0.248$ mixture is that of unpolluted stars, while all the others corresponds to various degrees of pollution by FRMS (see Chantereau et al. 2015). We stress that on the giant branches the chemical compositions are additionally affected by stellar evolution. We therefore give the initial composition that is encountered on the MS.

Table A.1. Mass fraction of key elements on the MS for several chemical compositions labeled by their helium mass fraction $Y$.

\begin{tabular}{lcccccccc}
\hline \hline Specie & $Y=0.248$ & $Y=0.260$ & $Y=0.270$ & $Y=0.300$ & $Y=0.330$ & $Y=0.370$ & $Y=0.400$ & $Y=0.600$ \\
\hline $\mathrm{H}$ & 0.751 & 0.739 & 0.729 & 0.699 & 0.669 & 0.629 & 0.599 & 0.399 \\
$\mathrm{He}$ & 0.248 & 0.260 & 0.270 & 0.300 & 0.330 & 0.370 & 0.400 & 0.600 \\
$\mathrm{C}$ & $9.4 \times 10^{-5}$ & $8.6 \times 10^{-5}$ & $8.0 \times 10^{-5}$ & $7.0 \times 10^{-5}$ & $6.2 \times 10^{-5}$ & $5.5 \times 10^{-5}$ & $5.1 \times 10^{-5}$ & $3.9 \times 10^{-5}$ \\
$\mathrm{~N}$ & $2.9 \times 10^{-5}$ & $8.9 \times 10^{-5}$ & $1.2 \times 10^{-4}$ & $1.8 \times 10^{-4}$ & $2.3 \times 10^{-4}$ & $2.8 \times 10^{-4}$ & $3.0 \times 10^{-4}$ & $4.0 \times 10^{-4}$ \\
$\mathrm{O}$ & $5.2 \times 10^{-4}$ & $4.7 \times 10^{-4}$ & $4.4 \times 10^{-4}$ & $3.8 \times 10^{-4}$ & $3.4 \times 10^{-4}$ & $2.9 \times 10^{-4}$ & $2.7 \times 10^{-4}$ & $1.7 \times 10^{-4}$ \\
$\mathrm{Na}$ & $1.1 \times 10^{-6}$ & $2.7 \times 10^{-6}$ & $3.3 \times 10^{-6}$ & $4.8 \times 10^{-6}$ & $5.7 \times 10^{-6}$ & $6.6 \times 10^{-6}$ & $7.1 \times 10^{-6}$ & $7.7 \times 10^{-6}$ \\
$\mathrm{Mg}$ & $3.6 \times 10^{-5}$ & $3.6 \times 10^{-5}$ & $3.6 \times 10^{-5}$ & $3.5 \times 10^{-5}$ & $3.5 \times 10^{-5}$ & $3.5 \times 10^{-5}$ & $3.4 \times 10^{-5}$ & $3.3 \times 10^{-5}$ \\
$\mathrm{Al}$ & $1.8 \times 10^{-6}$ & $2.4 \times 10^{-6}$ & $2.8 \times 10^{-6}$ & $3.7 \times 10^{-6}$ & $4.4 \times 10^{-6}$ & $5.2 \times 10^{-6}$ & $5.6 \times 10^{-6}$ & $7.4 \times 10^{-6}$ \\
\hline
\end{tabular}




\section{Appendix B: $Y$ difference from various colors}

In this appendix we show the determination of the maximum $Y$ difference in NGC 6752 using the colors (m410M-m814W) and (m467M-m814W).
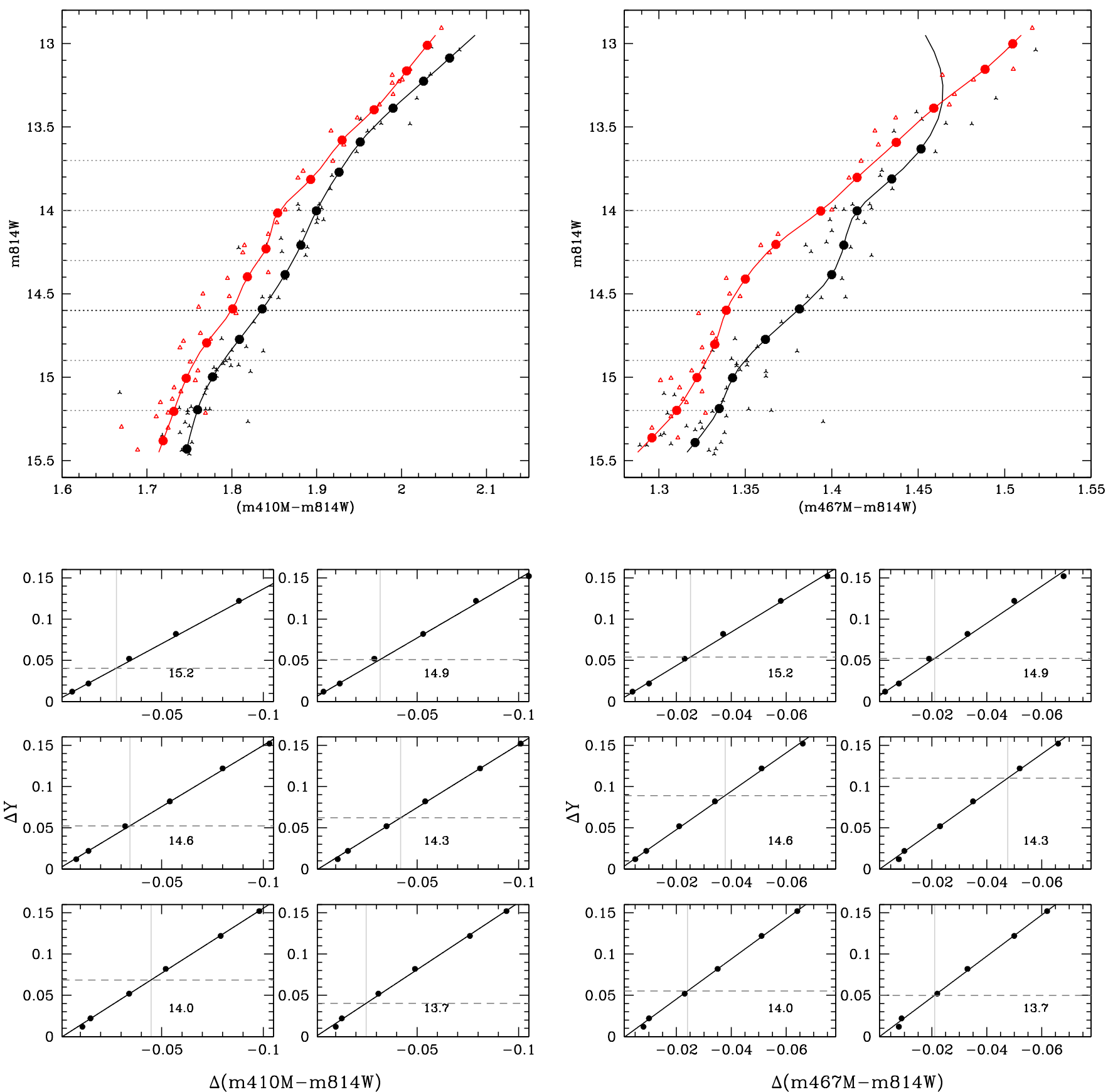

Fig. B.1. Same as Fig. 8 (top panels) and Fig. 9 (bottom panels) for a the colors (m410M-m814W) (left panels) and (m467M-m814W) (right panels). 